\title{
Fully resolved simulations of colliding monodisperse spheres in forced isotropic turbulence
}

\author{
By ANDREAS TEN CATE, JOS J. DERKSEN \\ LUIS M. PORTELA AND HARRY E. A. VAN DEN AKKER \\ Kramers Laboratorium voor Fysische Technologie, Delft University of Technology \\ Prins Bernhardlaan 6, 2628 BW Delft, The Netherlands
}

(Received 23 July 2002 and in revised form 28 July 2004)

Fully resolved simulations of particles suspended in a sustained turbulent flow field are presented. To solve the Navier-Stokes equations a lattice-Boltzmann scheme was used. A spectral forcing scheme is applied to maintain turbulent conditions at a Taylor microscale Reynolds number of 61 . The simulations contained between 2 and $10 \mathrm{vol} \%$ particles with a solid to fluid density ratio between 1.15 and 1.73. A lubrication force is used to account for subgrid hydrodynamic interaction between approaching particles. Results are presented on the influence of the particle phase on the turbulence spectrum and on particle collisions. Energy spectra of the simulations show that the particles generate fluid motion at length scales of the order of the particle size. This results in a strong increase in the rate of energy dissipation at these length scales and a decrease of kinetic energy at larger length scales.

Collisions due to uncorrelated particle motion are observed (primary collisions), and collision frequencies are in agreement with theory on inertial particle collisions. In addition to this, a large number of collisions at high frequencies is encountered. These secondary collisions are due to the correlated motion of particles resulting from shortrange hydrodynamic interactions and spatial correlation of the turbulent velocity field at short distances. This view is supported by the distribution of relative particle velocities, the particle velocity correlation functions and the particle radial distribution function.

\section{Introduction}

In industrial processes where solid materials are produced or handled, often dense slurries are processed under highly turbulent conditions. Turbulence is required to maintain the slurry suspended and well mixed. While operating under such conditions, phenomena such as breakage, agglomeration and segregation of particles can occur; these phenomena can be either desired or potential problems in the operation of these processes (Zwietering 1958; Wibowo \& Ng 2001).

One specific example is industrial crystallization, which deals with the production of solid crystals from a supersaturated liquid. The crystal suspension typically contains between 5 and 20 vol \% solids with an average particle size in the range of 100 to $1000 \mu \mathrm{m}$, and with a solid to liquid density ratio in the range of 1 to 2.5 . The Kolmogorov length scale typically is one order of magnitude smaller than the mean crystal size (Ten Cate et al. 2001). Under the action of the turbulent flow field, agglomeration (Hollander et al. 2001), abrasion, and fracture (Gahn \& Mersmann 
1999) take place. These phenomena have a strong influence on the performance of the crystallization process and hence on the resulting product, and make scale-up a non-trivial exercise.

In this paper we study turbulent solid-liquid suspensions under conditions that are comparable to those encountered in industrial crystallization processes (i.e. $d_{p}>\eta$, where $d_{p}$ is the particle diameter and $\eta$ the Kolmogorov length scale, and up to $10 \mathrm{vol}$ $\%$ solid phase). Strong hydrodynamic interactions between particles and fluid motion and strong particle-particle interactions are anticipated. We performed numerical simulations where the flow field around each particle is resolved and the motion of the solid and the fluid phase are fully coupled.

Much effort is currently invested in the development of efficient numerical schemes for the direct simulation of suspensions of solid spheres. A variety of different schemes are being developed based on finite difference or finite volume solution methods for the Navier-Stokes equations combined with efficient schemes for implementation of the solid particle boundary conditions (see e.g. Hu 1996; Hu, Patankar \& Zhu 2001; Pan \& Banerjee 1997; Patankar et al. 2000; Patankar 2001).

An alternative method for the direct simulation of suspensions is based on the lattice-Boltzmann method (see e.g. the review by Koch \& Hill 2001). The latticeBoltzmann method presents an efficient numerical scheme for solution of the NavierStokes equation on a regular cubic grid (Chen \& Doolen 1998). The approach was established and validated by Ladd $(1994 a, b)$. It has been used to study suspension problems with particles moving in the Stokes regime or at low but non-zero Reynolds numbers (e.g. sedimentation of large numbers of particles Ladd (1997, 2002), motion of spherical and non-spherical particles (Qi 1999; Aidun, Lu \& Ding 1998; Ding \& Aidun 2000). Both because of these promising results, and because we had previous experience with this numerical method, it was decided to use the lattice-Boltzmann method for our current study. However, the approach to simulate suspensions as presented in this paper differs from the methods presented by these authors on a number of points.

First, a major difference is that in the above simulations the flow field is laminar, while in our case we are interested in simulating a turbulent flow. To obtain meaningful statistical data, transient simulations are required with statistically stationary conditions. For this purpose, a turbulence forcing scheme, developed by Alvelius (1999), is applied to the lattice-Boltzmann scheme.

Secondly, both the type of lattice-Boltzmann scheme and the method to implement the no-slip particle boundary condition are different. The lattice-Boltzmann scheme used here is based on Eggels \& Somers (1995). This scheme explicitly treats higherorder terms of the lattice-Boltzmann equation, which improves the numerical stability of the scheme, compared to the standard BGK scheme (Chen \& Doolen 1998). The regular no-slip boundary condition used in lattice-Boltzmann simulations is the bounce-back method (Ladd 1994a). We do not use this method in our simulations, but instead employ an adaptive force-field technique to impose the no-slip particle boundary. This method is used by Derksen \& Van den Akker $(1999,2000)$ in large-eddy simulation (LES) studies of the turbulent flow in various applications and resembles a forcing-type boundary condition of Goldstein, Handler \& Sirovich (1993). In a previous study we compared our simulations with particle image velocimetry (PIV) experiments on a single sphere settling under gravity. That study demonstrated that an accurate representation of the sphere's motion and the associated flow field could be obtained at a resolution of approximately 8 gridpoints per particle diameter (Ten Cate et al. 2002). 
A vast body of literature exists that discusses the interactions between suspended particles and a turbulent flow field. The main issues treated in these studies are the motion and collisions of particles due to the turbulent flow field and the modification of turbulence due to the presence of a second phase.

A theoretical basis for the collision rate of small particles (i.e. $d_{p} \ll \eta$ ) suspended in a turbulent flow is provided by Saffman \& Turner (1956), for the purpose of describing agglomeration of droplets in atmospheric flows. Theoretical extensions of this work were proposed by Yuu (1984) and Kruis \& Kusters (1997) to improve the description of the particle motion in turbulent flow fields. Abrahamson (1975) presented a model to predict collision rates of particles with considerable inertia. These models have been used to interpret simulation results in a wide range of numerical studies on turbulent suspensions. In these studies the particles are typically much smaller than the resolution of the computational grid while Stokes drag is assumed to dominate the motion of the particles (Sundaram \& Collins 1997; Reade \& Collins 2000; Wang, Wexler \& Zhou 1998, 2000; Brunk, Koch \& Lion 1998).

Theoretical models that describe the modification of the turbulent flow field due to the presence of particles have been presented by e.g. Felderhof \& Ooms (1989), Ooms \& Jansen (2000), Yuan \& Michaelides (1992), Crowe (2000). Turbulence modification has been studied numerically in two-way coupled simulations of isotropic turbulence, e.g. Squires \& Eaton (1990), Elghobashi \& Truesdell (1993) and Boivin, Simonin \& Squires (1998).

In contrast with our approach (i.e. $d_{p}>\eta$ ), the above mentioned studies focus on systems with small particles with a low volume fraction of the solid phase $\left(O\left(10^{-4}\right.\right.$ $\left.10^{-6}\right)$ ). Although the regime in our simulation is much different from that in the above studies, some of the concepts used in or obtained from these studies are used in this paper for qualitative comparison and discussion of the trends in our results.

The objectives of this paper are:

(i) presentation of our method for simulation of fully resolved dense suspensions;

(ii) demonstration of the use of a spectral forcing scheme in lattice-Boltzmann simulations for generation of sustained turbulence with pre-defined conditions;

(iii) discussion of the modification of the turbulent energy spectrum under influence of suspended particles;

(iv) interpretation of the collision behaviour and the relative particle motion observed in dense turbulent solid-liquid suspensions.

These objectives are reflected in the organization of the paper; after the presentation of our numerical approach $(\S 2)$, the settings and results for a single-phase isotropic turbulent simulation are presented in $\S 3$. In $\S 4$ the influence of the particle phase on the turbulent fluid kinetic energy spectra is discussed. In $\S 5$, the behaviour of the particle phase is discussed in relation to the theoretical work of Wang et al. (2000) and Reade \& Collins (2000). This discussion focuses on the influence of the turbulent flow field and the short-range hydrodynamic interactions, on the distribution of relative velocities, particle accumulation effects and particle collision rates. The paper ends with a summary and conclusions in $\S 6$.

\section{Set-up of the direct numerical simulation}

\subsection{The lattice-Boltzmann method}

A lattice-Boltzmann scheme has been used for simulation of the fluid flow. This scheme is based on a microscopic model for fluid behaviour. Our aim is to do highresolution simulations on large computational grids. The lattice-Boltzmann method 
is well suited to this goal, since it only involves local operations. As a result, parallel implementation of the method is straightforward and the computational effort scales practically linearly on parallel computer platforms.

The fluid is represented by mass that propagates at discrete time steps on an equidistant grid (lattice). Collision rules that guarantee conservation of mass and momentum are applied on the mass arriving at a grid node. The collision process is represented by the lattice-Boltzmann equation (LBE)

$$
n_{i}\left(\boldsymbol{x}+\boldsymbol{c}_{i} \Delta t, t+\Delta t\right)=n_{i}(\boldsymbol{x}, t)+\Gamma_{i}(\boldsymbol{n}) .
$$

Due to collision an amount of mass $n_{i}$, aligned in the direction $\boldsymbol{c}_{i}$ (with $i=1, M$ ) at position $x$ and time $t$, is updated with $\Gamma_{i}$ after which it propagates to the position $\boldsymbol{x}+\boldsymbol{c}_{i} \Delta t$ at the next time step $t+\Delta t$. Here, $\boldsymbol{c}_{i}$ is the set of discrete velocities with which mass moves on the grid, and $\Gamma_{i}$ is the collision operator that depends on all masses, i.e. the vector $\boldsymbol{n}$, involved in the collision step. It is common practice to define the grid distance $\Delta x$ in terms of lattice units and the time increment $\Delta t$ in terms of discrete time steps. The grid distance and time step are defined as $\Delta x=1[l u]$ and $\Delta t=1[t s]$. Throughout this paper the units are accordingly defined in lattice units $[l u]$ for length, time steps $[t s]$ for time and arbitrary units of mass $[\mathrm{m}]$.

Different approaches have been employed to solve equation (2.1) (see e.g. Ladd 1994a; Chen \& Doolen 1998; Rothman \& Zaleski 1997). In this work we use a scheme developed by Eggels \& Somers (1995). With this scheme the following continuity and momentum equations for the fluid are recovered:

$$
\begin{gathered}
\frac{\partial \rho}{\partial t}+\nabla \cdot \rho \boldsymbol{u}=0, \\
\frac{\partial \rho \boldsymbol{u}}{\partial t}+\nabla \cdot \rho \boldsymbol{u} \boldsymbol{u}=-\nabla p+\nabla \cdot \rho v\left[\nabla \boldsymbol{u}+(\nabla \boldsymbol{u})^{T}\right]-\nabla\left(\frac{1}{2} \rho \nu \nabla \cdot \boldsymbol{u}\right)+\boldsymbol{F},
\end{gathered}
$$

where $p(\boldsymbol{x}, t)$ is the pressure and $v$ is the kinematic fluid viscosity. In the limit of low Mach numbers (i.e. $\boldsymbol{u} \ll c_{s}$, the speed of sound of the lattice-Boltzmann scheme), the divergence terms in equations (2.2) and (2.3) become negligibly small and the set of equations becomes equivalent to the continuity and Navier-Stokes equations for incompressible fluid flow. The body force $\boldsymbol{F}(\boldsymbol{x}, t)$ in equation (2.3) imposes a local force per unit volume. This force is used in our simulations for two goals: first for the generation of sustained turbulent conditions and secondly for the implementation of the particle boundary conditions.

\subsection{Turbulence forcing}

In a typical turbulent flow, kinetic energy is produced at large scales, and dissipated at the small scales. By forcing the fluid at small wavenumbers, the fluid is set in motion at large length scales. At sufficiently large Reynolds numbers, i.e. at a sufficiently large separation between production and dissipation scales, the small-scale structure exhibits universal characteristics of turbulent fluid motion independently of the means of power input. Schemes for forcing turbulent conditions have been developed by a number of authors, e.g. Eswaran \& Pope (1988), and more recently by Overholt \& Pope (1998) and Alvelius (1999).

For application of these schemes in numerical simulations, (pseudo) spectral methods for the solution of the Navier-Stokes equations are the natural choice, since such numerical schemes are defined in wavenumber space, as is the forcing scheme. However, the forcing scheme is not necessarily associated with spectral simulation methods. In our work we use the algorithm developed by Alvelius (1999) for forcing 


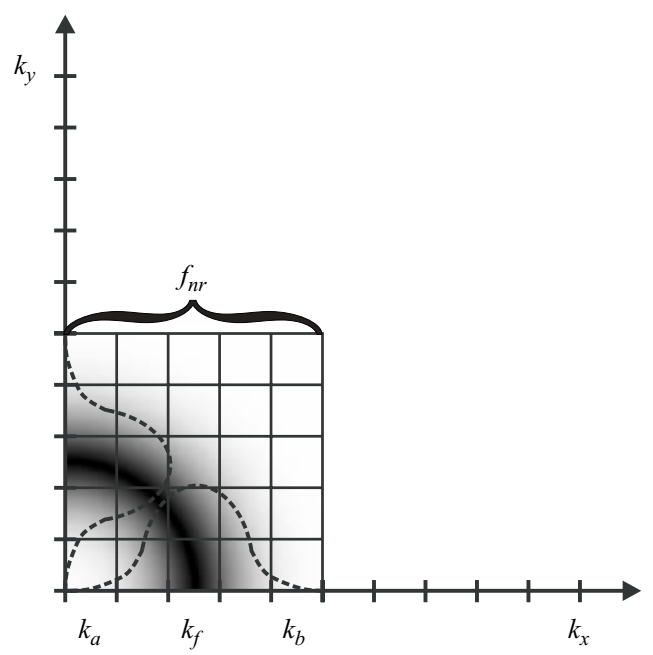

FiguRE 1. Two-dimensional representation of the forcing distribution in the Fourier domain. Dotted curves indicate the Gaussian distribution along the $x$ - and $y$-axes, the shading indicates the Gaussian distribution in the two-dimensional plane.

the flow in the lattice-Boltzmann scheme. The turbulent forcing signal is generated in Fourier space and, after computing the inverse Fourier transform, is applied in the physical domain (i.e. in equation (2.3) via the body force $\boldsymbol{F}(\boldsymbol{x}, t)$ ). The force is defined as a divergence-free white noise signal, fluctuating randomly in time and space. The white noise character ensures that the forcing signal is uncorrelated with any time scale of the turbulent flow field. Alvelius shows that during one discrete time step, the volume-averaged power input $P$ consists of the sum of two contributions,

$$
P=P_{1}+P_{2}=\frac{1}{2} \overline{f_{k} f_{k}} \Delta t+\overline{f_{k} u_{k}},
$$

the force-force correlation and the velocity-force correlation, respectively (the body force per unit volume, $F_{k}$ and acceleration per unit mass, $f_{k}$ are related by $F_{k} \equiv \rho f_{k}$ ). The overbar indicates volume-averaging. The force is placed in the discrete Fourier space in a cubic cell with sides $f_{n r}$. The forcing intensity is distributed over the wavenumbers as a Gaussian distribution, active on the interval $\left[k_{a}, k_{b}\right]$, with the maximum intensity at a desired wavenumber $k_{f}$ (see figure 1).

The power input $P_{1}$ is fixed via the Gaussian distribution of the force in Fourier space. $P_{2}$ introduces an uncontrolled power input that influences the turbulent kinetic energy and can generate substantial fluctuations. The method of Alvelius (1999) allows one to choose the force such that the contribution of $P_{2}$ is zero at each time step. This results in a much smoother time development of the turbulent kinetic energy. Using this option requires the Fourier transform of the velocity field every time step. It was not used in the current study because of this additional computational cost.

Alvelius chose the distribution of the power input to be narrowly concentrated around the forcing wavenumber $k_{f}$, which can cause the total power input to rise up to 1.5 times $P_{1}$. In the simulations presented in this paper we chose to distribute the power input more evenly over the wavenumbers in the forcing interval $\left[k_{a}, k_{b}\right]$ by increasing the width of the Gaussian distribution. This is controlled by a concentration parameter $c$, which was set to 1.0 in our simulation, as opposed to the value of 0.01 that is used by Alvelius (1999). In this way we found that the large increase in power input due to $P_{2}$ could be effectively suppressed. 
For a further enhancement of the computational efficiency, rather than generating a new force field each time step, 120 force fields were generated prior to the simulations. These were imposed on the fluid domain in random order during the simulations. In this way, a forcing signal was generated that sufficiently resembled a white noise signal.

\subsection{Solid particle boundary conditions}

Different simulation strategies are being developed for the direct simulation of freely moving particles. The choice of the method for implementation of the solid particle boundary condition is generally strongly related to the choice of discretization method of the Navier-Stokes equations.

One approach is to solve the flow field on an unstructured computational grid, the shape of which is determined by the geometry of the cluster of freely moving particles. During the simulation the quality of the computational mesh is checked and the mesh is frequently updated, based on the changing geometry of the cluster of particles. With this method, Hu (1996) performed two-dimensional simulations of sedimentation of a few hundred cylinders in a channel, while a more detailed analysis and results on three-dimensional simulations of moving spheres are presented in $\mathrm{Hu}$ et al. (2001).

An alternative method is to impose the boundary condition of freely moving particles using a fixed structured grid. One advantage of this type of method is that it does not require remeshing of the computational grid. A number of different approaches are currently being applied.

One approach in this category is to use a local force on the computational grid to obtain no-slip boundary conditions. The force ensures that the flow field is locally in agreement with the particle boundary conditions. Examples of this method are given by Goldstein et al. (1993) and by Pan \& Banerjee (1997). The latter used this method to simulate both steady and moving particles in DNS of a turbulent channel. They chose to apply a force on each of the grid points that is covered by the simulated spheres. In this way the fluid on the inside of the sphere is forced to behave as a solid body.

Another approach in which the covered fluid nodes are treated as a solid body is given by the Lagrange-multiplier/fictitious domain method. A recent example of this method is given by Patankar et al. (2000) while ongoing developments are presented in Patankar (2001). The recent developments mainly focus on obtaining a fundamental understanding of the formulation of the forcing term and on increasing computational efficiency.

A recent paper by Lai \& Peskin (2000) presents a formal second-order-accurate forcing scheme for the solid boundary condition, based on a finite difference discretization of the Navier-Stokes equations. Lai \& Peskin (2000) define the surface of a cylinder by a number of discrete points that are used to impose a force on the fluid on the interior and exterior grid points adjacent to the surface. As a result, the fluid on the inside of the cylinder develops an internal flow.

In lattice-Boltzmann applications, the natural choice for a no-slip boundary condition is the bounce-back method. In this approach fluid mass that propagates along a link that crosses the surface of the particle bounces back halfway between the grid nodes and returns towards the bulk fluid domain. The momentum change at bounce-back is associated with the force acting on the particle. With this method, curved objects become staircase shaped due to the discrete cut of the links between nodes on the cubic grid. A number of adaptations of the bounce-back technique 


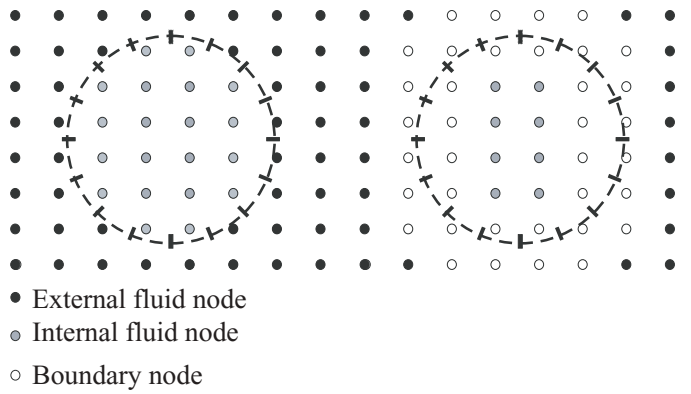

FigURE 2. Representation of a sphere on a cubic grid. The tick marks indicate control points at the sphere surface. The left-hand circle indicates the division between interior and exterior fluid nodes. The right-hand circle indicates the nodes from which the velocity is interpolated and to which the forces that impose the boundary condition are applied.

have been suggested to improve the representation of curved boundaries (e.g. Chen, Teixeira \& Molvig 1998a; Rohde, Derksen \& Van den Akker 2002; Mei, Luo \& Shyy 1999; Verberg \& Ladd 2002; Bouzidi, Firdaouss \& Lallemand 2001). In most lattice-Boltzmann studies of suspended particles (e.g. Ladd 1994a,b; Behrend 1995; Qi 1999; Aidun et al. 1998) bounce-back-type boundary conditions are applied.

We use an alternative approach to obtain a no-slip boundary at the surface of the particle. In our current simulations we apply a forcing scheme to impose the boundary conditions which is based on the method of Goldstein et al. (1993) and which has been adapted for our lattice-Boltzmann scheme. By applying an appropriately chosen force to the fluid domain via the body force term $\boldsymbol{F}$ (equation (2.3)), the fluid velocity near the surface of the solid object is forced to the velocity of the solid surface, which effectively fulfils the no-slip boundary condition.

In our simulations, the sphere surface is represented by a set of control points, placed at the sphere's surface, evenly spaced at a distance apart somewhat smaller than the grid spacing (see figure 2). The surface velocity $\left(\boldsymbol{u}_{s}\right)$ is given by the sum of the translational and rotational velocity components

$$
\boldsymbol{u}_{s}(t)=\boldsymbol{u}_{p}(t)+\boldsymbol{\Omega}_{p}(t) \times \boldsymbol{r}_{p}
$$

where $\boldsymbol{u}_{p}$ and $\boldsymbol{\Omega}_{p}$ are the translational and angular velocity of the particle and $\boldsymbol{r}_{p}$ is the position of the control point relative to the centre of the sphere $\left(\boldsymbol{r}_{p}=\boldsymbol{x}_{p}-\boldsymbol{x}_{c}\right)$. The fluid velocity at a control point is determined via interpolation of the velocity from the surrounding grid nodes. The deviation between the desired velocity $\boldsymbol{u}_{s}$ and the interpolated fluid velocity $\boldsymbol{u}$ is used to compute the force that is applied to the fluid. This deviation is calculated by

$$
\boldsymbol{d}(t)=\boldsymbol{u}_{s}(t)-\sum_{j} I\left(\boldsymbol{r}_{j}\right) \boldsymbol{u}_{j}(t)
$$

where $\boldsymbol{u}_{j}$ is the fluid velocity at grid node $j$ and $I\left(\boldsymbol{r}_{j}\right)$ is the set of interpolation coefficients, which is a function of the position $\boldsymbol{r}_{j}$ of the control point with respect to the surrounding grid nodes. The same interpolation coefficients are used for projecting the force from the control point onto the surrounding grid nodes. As indicated in figure 2, the grid nodes on the outside as well as on the inside of the sphere are included in interpolation and projection. In previous applications, secondorder Lagrange interpolation was used (Derksen \& Van den Akker 1999). In our current simulations we use first-order or linear interpolation, to ensure that only 
the neighbouring grid nodes are affected, rather than neighbour and next-neighbour nodes. The results obtained with first-order interpolation were comparable with the second-order scheme.

The forces $\boldsymbol{F}_{j}$ acting at grid node $j$ are updated via

$$
\boldsymbol{F}_{j}(\boldsymbol{x}, t)=\alpha \boldsymbol{F}_{j}(\boldsymbol{x}, t-\Delta t)+\beta I\left(\boldsymbol{r}_{j}\right) \frac{\rho \boldsymbol{d}(t)}{\Delta t} .
$$

Equation (2.7) is a control scheme that is characterized by the parameters $\alpha$ and $\beta$. At each time step the force $\boldsymbol{F}_{j}(\boldsymbol{x}, t-\Delta t)$ that is present on the grid from the previous time step is updated proportionally to the deviation of the local velocity. When the deviation is small, the change of the force imposed at the previous time step will also be small and the force remains practically constant. The dynamic action, accuracy, and stability of the control scheme are determined by the parameters $\alpha$ and $\beta$ and the topology of the set of control points. The parameters $\alpha$ and $\beta$ were determined empirically as 0.95 and 1.8 respectively. With this choice of parameters a small deviation will always be present and the force will be updated each time step. It was observed in simple test cases that the no-slip boundary condition obtained with this scheme is of comparable quality to the boundary condition obtained with the bounceback rule for the lattice-Boltzmann scheme. The adaptive force field scheme was preferred over the bounce-back scheme because of the ease of placing the boundary conditions at arbitrary locations on the grid.

After updating the forces at the grid nodes, the hydrodynamic force and torque on the sphere are calculated. The hydrodynamic force and torque on the particle are determined from

$$
\begin{gathered}
\widehat{\boldsymbol{F}}_{p}=\Delta V \sum_{j} \boldsymbol{F}_{j}(\boldsymbol{x}, t), \\
\widehat{\boldsymbol{T}}_{p}=\Delta V \sum_{j} \boldsymbol{r}_{p} \times \boldsymbol{F}_{j}(\boldsymbol{x}, t),
\end{gathered}
$$

where $\Delta V=\Delta x^{3}$, the volume of the grid cell. The hat symbol is used to indicate force per particle. The summation is over all boundary nodes, both inside and outside the sphere.

\subsection{Internal fluid nodes}

The equation of motion for translation and rotation of the particles is integrated based on the hydrodynamic forces acting on the particles. The adaptive force-field scheme requires fluid mass on the grid nodes occupied by the solid object (see figure 2). An advantage is that a node that shifts from the inside of the object into the exterior already contains fluid mass and the scheme will be inherently mass-conservative (similar to Ladd 1994b). One drawback is that the internal fluid inertia influences the motion of the sphere via a contribution to $\widehat{\boldsymbol{F}}_{p}$ and $\widehat{\boldsymbol{T}}_{p}$ (see equations (2.8) and (2.9)).

This problem has been recognized by a number of authors who have suggested different solutions. Ladd (1994b) suggests integrating the equation of motion with an effective particle mass. This is a fair approximation for systems with large solid/fluid density ratios but for solid particles in liquid with a density ratio typically between 1 and 2, numerical instabilities may occur when integrating the equation of motion this way. Other authors have proposed methods to remove the internal mass, e.g. Aidun \& Lu (1995), Aidun et al. (1998) and Heemels (1999), but these methods cannot be applied in combination with the adaptive force-field technique. Qi (1999) allows particles to have internal mass but adds a force term (similar to Aidun et al. 
1998) when computing the hydrodynamic force on the particle, to compensate for non-physical fluctuations in the hydrodynamic force $\boldsymbol{F}_{p}$ that arise from nodes entering or leaving the interior of a particle.

We propose the following straightforward correction procedure. The force for the boundary condition is placed on the grid nodes both on the inside and the outside of the sphere. Hence, the total force that acts on the sphere, as calculated with equation (2.8), is a sum of the internal and external contribution to the force:

$$
\widehat{\boldsymbol{F}}_{p, t o t}=\widehat{\boldsymbol{F}}_{p, i n t}+\widehat{\boldsymbol{F}}_{p, e x t} .
$$

When integrating the equation of motion, the only physical contribution to the sphere's motion is due to the external flow field, $\widehat{\boldsymbol{F}}_{p, \text { ext }}$.

The fluid motion inside the particles is developing freely throughout a simulation in response to the forces $\boldsymbol{F}_{j}$ and generally follows the motion of the particle closely. To calculate the contribution of $\widehat{\boldsymbol{F}}_{p, \text { int }}$, we calculate the change in momentum of the internal fluid between successive time steps with

$$
\widehat{\boldsymbol{F}}(t)_{p, \text { int }}=\frac{1}{\Delta t}\left[\iiint_{V_{\text {int }}} \rho \boldsymbol{u}(\boldsymbol{x}, t) \mathrm{d} V-\iiint_{V_{\text {int }}} \rho \boldsymbol{u}(\boldsymbol{x}, t-\Delta t) \mathrm{d} V\right]
$$

where the volume integral is taken over $V_{\text {int }}$, the volume occupied by the nodes in the interior of the particle (see figure 2). The external force is then determined by subtracting $\widehat{\boldsymbol{F}}(t)_{p, \text { int }}$ from $\widehat{\boldsymbol{F}}(t)_{p, \text { tot }}$. A similar correction procedure is applied for the torque.

\subsection{Hydrodynamic radius}

The next issue that needs to be addressed is the effective radius at which a particle is implemented. By placing a sphere on a cubic grid, the shape is approximated by imposing the boundary condition on a cubic grid. The interpolation-extrapolation procedure extends outside the exact location of the sphere surface and hence the measured drag force will differ from the force on an ideal sphere of the same size.

Ladd (1994b) compared simulations of the low Reynolds number flow through a fully periodic array of spheres with the analytical solution to this problem (Hasimoto 1959). Via the analytic solution the effective radius of the sphere could be calculated. It was found that the effective or hydrodynamic radius differed from the input radius by on the order of 0.5 to 1 lattice spacing. This approach further demonstrated that the hydrodynamic radius varies with viscosity in a non-physical manner. To overcome this effect, Ladd (1994b) suggested using the analytical solution as a calibration method, by calculating the sphere's hydrodynamic radius prior to a suspension simulation.

This non-physical dependence of the drag force is also reported by Rohde et al. (2002), who performed simulations with an improved bounce-back boundary scheme. A possible reason for this dependence is given by He et al. (1997), who demonstrated analytically that in bounce-back methods for lattice-Boltzmann schemes, the exact location of the no-slip condition is a function of viscosity. However, it is not only an artifact of bounce-back-type boundary conditions, since a similar dependence is also observed with our adaptive force-field approach.

For particles with large input radii, a variation in the effective radius of the order of 0.5 to 1.0 grid spacing is not of great importance. However, for reasons of computational efficiency, if one seeks to perform simulations at a relatively low resolution, a calibration of the hydrodynamic radius is required. We found in a previous study (Ten Cate et al. 2002) that by calibrating the particle radius via the 
analytical expression of Hasimoto (1959), the transient motion of a single sphere settling in a closed tank could be predicted at an accuracy of approximately $1 \%$ to $5 \%$ with respect to the maximum sedimentation velocity. Although the calibration was performed in the creeping flow regime, the sedimentation experiment was mimicked accurately at Reynolds numbers in the range between 1.5 and 32 . The calibration procedure thus offers a reasonable method to estimate and account for the inaccuracy of the boundary condition, even when the particles in the actual case are moving at non-zero Reynolds numbers.

\subsection{Short-range interactions}

The flow field that surrounds the particles is simulated explicitly and hydrodynamic interactions between particles are inherently present. However, as two particles approach, one should guarantee that hydrodynamic effects at short distances are present, and one should allow particles to collide. For treatment of these short-range interactions between particles several procedures are available in the literature. An overview can be found in $\mathrm{Hu}$ et al. (2001). A procedure applied in unstructured mesh simulations of moving particles is to remesh the grid inbetween two approaching particles up to a minimal resolution. In such simulations a minimal separation between the particles is set at which collisions occur. A drawback of such a procedure is that it is difficult to control the computational effort. In simulations of suspensions using structured grids, remeshing of the flow field in between two particles is not possible. A number of different procedures are used in these types of simulations. Generally, to keep the particles separated either hard-sphere collision detection or a strong short-range repulsive force is applied. To estimate the short-range viscous forces exerted on the particles due to the squeezing motion of the fluid in the gap between the particles, a formulation of the lubrication force can be applied. Maury (1997) presents a formal mathematical formulation to apply a lubrication force model to compute the motion of large numbers of arbitrarily shaped particles whose motion is dominated by lubrication forces.

In our current simulations we use a collision detection algorithm according to Chen, Kontomaris \& McLaughlin (1998b) to prevent particles overlapping and to detect and record particle-particle collisions. When two particles are separated by a distance smaller than the grid spacing, the motion of the interstitial fluid will not be captured due to lack of spatial resolution.

When two particles are close to each other, the grid nodes in between the two particles will contain force contributions from the boundary conditions of both particles. In such a case, overlap of forcing on a grid node occurs. The force applied to the grid node where overlap occurs cannot be identified with any of the two particles in particular and this force is set to zero. The fluid motion in the proximity of this grid node is dominated by the motion of the particles and the velocity on the released node will adopt a value that corresponds to the motion of its surrounding flow field.

At the same time, the force that is exerted on the particles due to the interstial fluid will be under-predicted. To ensure that the hydrodynamic forces between two approaching particles are present when two particles are separated by less then one grid spacing, a lubrication force is applied in a manner similar to Ladd (1997), who proposed calculating the missing part of the hydrodynamic forces explicitly, using the leading-order term of the analytic expression for the lubrication force (e.g. Kim \& Karrila 1991; Crowe, Sommerfeld \& Tsuji 1997). This force is calculated for pairs of particles that are closer than a threshold separation $\Delta_{0}$ by taking the difference of 
the force at distance $s$ and $\Delta_{0}$ (set to two lattice spacings in our simulations),

$$
\widehat{\boldsymbol{F}}_{i j}^{l u b}=\frac{-3 \pi \rho v r_{p}^{2}}{2}\left(\frac{1}{s}-\frac{1}{\Delta_{0}}\right) \widehat{\mathbf{x}}_{i j} \widehat{\mathbf{x}}_{i j} \cdot\left(\boldsymbol{u}_{i}-\boldsymbol{u}_{j}\right)
$$

where $s$ is the width of the gap between particles $i$ and $j, \boldsymbol{u}_{i}$ and $\boldsymbol{u}_{j}$ are the particle velocities and $\widehat{\mathbf{x}}_{i j}=\left(\boldsymbol{x}_{i}-\boldsymbol{x}_{j}\right) /\left|\boldsymbol{x}_{i}-\boldsymbol{x}_{j}\right|$ is the vector with unit length connecting the centres of the two particles.

This force is complementary to the forces that act on the particles due to the external flow field and is therefore added to the force calculated by imposing the boundary condition. Ladd \& Verberg (2001) chose to implement the lubrication force according to equation (2.12) on a particle pairwise basis. In suspensions at high volume concentrations or when accumulation of particles occurs, a particle may have multiple interactions with a number of surrounding particles. In our simulations, the lubrication forces are calculated for the interaction between each particle and all of its neighbouring particles at each time step.

If the lubrication force (2.12) is the only force that dictates the motion of two approaching particles, then the relative velocity will be fully dissipated and particles will always come to a halt at a finite separation. Brunk et al. (1998) present a theoretical study on the coagulation of colloidal particles in a turbulent flow field, where the role of Van der Waals forces and lubrication forces is evaluated. The authors state that colloidal particles can only collide under the action of a lubrication force when some attractive force such as the Van der Waals force is present.

However, for two approaching particles with sufficient inertia (as opposed to colloidal particles), the final separation can become so small that the continuum description of the lubrication model breaks down (i.e. when the gap becomes of the order of the particle surface roughness (Joseph et al. 2001) or of the order of the molecular mean free path (Sundararajakumar \& Koch 1996) and particles have practically established contact. In the presence of turbulent shear forces, the approach of particles can be further enhanced by the shear flow field and particle collisions are likely to occur.

Experiments also demonstrate that particles that move in a liquid and have sufficient inertia will rebound when colliding with a bottom (Gondret et al. 1999; Gondret, Lance \& Petit 2002) or vertical wall (Joseph et al. 2001) and a moment of contact has occurred. In our previous work on the simulation of a single settling sphere (Ten Cate et al. 2002) a comparison between simulation results and experimental data showed that application of a lubrication force adds a repulsive force that improves the description of the wall approach. However, it was also observed that without the use of a threshold value, the application of a lubrication force tends to postpone the moment of contact between the particle and the bottom wall for an unrealistically long time.

This behaviour is caused by the singularity in the lubrication model at zero separation. Different authors have used a threshold separation up to which the lubrication force is applied to overcome this singularity. In the analysis of their experimental results, Joseph et al. (2001) show that trends in their experimental data are captured when the lubrication force is calculated up to a threshold value that corresponds to the surface roughness of the particles (typically of the order of $10^{-5}$ times the particle diameter). Brunk et al. (1998) used a threshold separation of the order of $10^{-4}$ times the particle diameter, at which the attractive Van der Waals forces have become the dominating force and collisions have become inevitable. 
When performing turbulent suspension simulations with a lubrication force model without a threshold, we found that the singularity of the lubrication model caused particle pairs to become trapped in an unphysical pairwise oscillating motion. In our simulations we therefore used a dynamic threshold up to which the lubrication force is active. The gap between two approaching particles can be travelled in one time step when the relative velocity is equal or larger than the separation $s / \Delta t$. Therefore, when $s / \Delta t$ becomes of the order of the pair relative velocity, the contribution due to the lubrication force is set to zero:

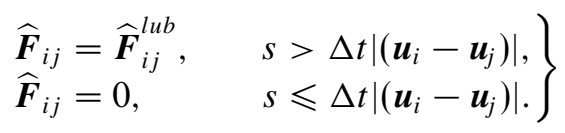

Conversely, as two particles separate, the lubrication force remains zero until the post-collision separation of the particles has become larger than their relative velocity. Since the particle relative velocity is small, the accuracy of this rule is expected to be comparable to the threshold rules described above. The threshold can be evaluated from the relative velocity distributions obtained from the simulations. Based on the r.m.s. relative velocity from these distributions we find that the threshold becomes approximately $0.01[l u]$, which is roughly $10^{-3}$ times the particle diameter.

\subsection{Simulation procedure}

During the simulations the above steps are carried out sequentially at each time step. The beginning of a sequence consists of integration of the fluid and particle fields. The first step is integration of the fluid velocity field from the previous time step $t-\Delta t$ to $t$ based on a lattice-Boltzmann step. After this update, flow field properties such as $\boldsymbol{u}(t)$ are available. After updating the fluid velocity field, the particle position and velocity are updated. The positions and translational and angular velocities are integrated using an explicit forward Euler integration scheme,

$$
\begin{aligned}
\boldsymbol{X}_{i}^{t} & =\boldsymbol{X}_{i}^{t-\Delta t}+\boldsymbol{V}_{i}^{t-\Delta t} \Delta t, \\
\boldsymbol{V}_{i}^{t} & =\boldsymbol{V}_{i}^{t-\Delta t}+\frac{1}{2 M_{p}}\left(\widehat{\boldsymbol{F}}_{i}^{t-\Delta t}+\widehat{\boldsymbol{F}}_{i}^{t-2 \Delta t}\right) \Delta t, \\
\boldsymbol{\Omega}_{i}^{t} & =\boldsymbol{\Omega}_{i}^{t-\Delta t}+\frac{1}{2 I_{p}}\left(\widehat{\boldsymbol{T}}_{i}^{t-\Delta t}+\widehat{\boldsymbol{T}}_{i}^{t-2 \Delta t}\right) \Delta t,
\end{aligned}
$$

where $\widehat{\boldsymbol{F}}_{i}$ and $\widehat{\boldsymbol{T}}_{i}$ are the sum of forces and torques acting on particle $i$ with mass $M_{p}$ and moment of inertia $I_{p}$. The particle mass and moment of inertia in this scheme are determined based on the particle density and the particle radius that was obtained from the hydrodynamic radius calibration procedure. Both the forces and torques are time-averaged over the previous two time steps to reduce fluctuations (see also e.g. Aidun et al. 1998).

After updating the particle positions and velocities, the force field of the particle boundary condition is updated to time $t$ and the force $\widehat{\boldsymbol{F}}_{p}^{t}$ and torque $\widehat{\boldsymbol{T}}_{p}^{t}$ of each particle are calculated. At the new positions the change of the internal momentum of the particles is calculated and forces and torques are updated to $\widehat{\boldsymbol{F}}_{p, \text { ext }}^{t}$ and $\widehat{\boldsymbol{T}}_{p, \text { ext }}^{t}$. After calulation of the lubrication forces, the total sum of forces acting on each particle is again computed.

After executing these steps the force field for forcing the turbulence is computed or retrieved from file. At this stage a complete time sequence has been executed, the time step is updated to the next time step and a new lattice-Boltzmann flow field update is carried out. 


$\begin{array}{cl}\text { Input parameters } & \text { Value } \\ v_{*}^{\prime} & 0.02 \\ P_{\text {input }} \times 10^{8} & 6.25 \\ P_{1} \times 10^{8} & 7.19 \\ \eta & 1.2 \\ v \times 10^{3} & 5.06\end{array}$

TABLE 1. Parameter settings for the single-phase turbulent simulations.

\section{Single-phase isotropic forced turbulence}

\subsection{Definition of turbulent conditions}

Homogeneous isotropic turbulent simulations were carried out on a $256^{3}$ computational grid with periodic boundary conditions. Simulations were performed from a developed flow field and were continued for 40000 time steps. The turbulent simulation is characterized by a number of a priori defined parameters given in table 1 . The forcing is defined by setting a characteristic root mean square velocity $\left(v_{*}^{\prime}\right)$ and a characteristic forcing length scale $l_{*}$. The value of $v_{*}^{\prime}$ was chosen such that velocities in the simulation will remain well below the speed of sound of the lattice-Boltzmann scheme $\left(c_{s}=\frac{1}{2} \sqrt{2}\right)$. The central forcing wavenumber determines the forcing length scale: $k_{f}=2 \pi / l_{*}$, which was set to $l_{*}=n_{x} / 2$. The power input is determined by the equilibrium between production and dissipation, resulting in $P_{\text {input }}=v_{*}^{\prime 3} / l_{*}$. In table 1 both $P_{\text {input }}$ and the effective power input $P_{1}$ (from equation (2.4)) are given. The deviation results from the error made by discretizing the power on a cube in the Fourier domain with sides of three points. The final parameter that is defined is the kinematic viscosity, which is set by choosing a Kolmogorov length scale. In a statistical equilibrium, the rate of energy dissipation balances the power input, i.e. $P=\epsilon$, and the Kolmogorov length and time scales are set a priori via $\eta=\left(v^{3} / \epsilon\right)^{1 / 4}$ and $\tau_{k}=(v / \epsilon)^{1 / 2}$. The Kolmogorov length scale was chosen as $1.2[l u]$ after a number of considerations: (i) the Kolmogorov length scale is chosen small enough to obtain a separation of length scales between the microscopic length scale $\eta$, the particle size $d_{p}$ and the integral scale of the turbulence $\Lambda$, (ii) in direct simulations of forced isotropic turbulence, a rule of thumb for adequate resolution of the flow field is $k_{\max } \eta>1$ (see e.g. Sundaram \& Collins 1997), where $k_{\max }=\pi / \Delta x$ is the largest resolved wavenumber. With $\eta=1.2$ being roughly four times the minimum value of the rule of thumb we chose to stay on the safe side of this requirement. The large solid particles are expected to induce velocity gradients at their surface. With the choice of a relatively large Kolmogorov length, the occurrence of prohibitively strong local velocity fluctuations or velocity gradients can be prevented. This will help to ensure that the gradients in the flow field induced by the particles are resolved instead of overwhelmed by the action of the turbulent flow field.

The turbulent Reynolds number that characterizes the simulations will follow these settings. By choosing a rather large value for the Kolmogorov length scale compared to typical simulations of isotropic turbulence reported in the literature (Moin \& Mahesh 1998), the turbulent flow field will attain a moderate Reynolds number.

\subsection{Results of single-phase turbulence}

In table 2, a number of properties are presented that characterize the simulation. Flow field data were stored every 4000 time steps (approximately 2 integral time scales) 


$\begin{array}{lclcc}\text { Property } & \text { Value } & \text { Scale } & \Lambda_{i} & T_{i} \\ K \times 10^{4} & 4.28 & \Lambda_{K}, T_{K} & 74.28 & 6598 \\ u^{\prime} & 0.017 & \Lambda_{E}, T_{E} & 37.71 & 2245 \\ \epsilon_{l B} \times 10^{8} & 6.48 & \Lambda_{f}, T_{f} & 42.47 & 2515 \\ \epsilon_{E} \times 10^{8} & 6.42 & \Lambda_{g}, T_{g} & 22.17 & 1313 \\ \eta & 1.19 & & & \\ \eta_{\min } & 0.45 & & & \\ \tau_{k} & 279 & & & \\ \lambda & 18.27 & & & \\ R e_{\lambda} & 60.98 & & & \end{array}$

TABLE 2. Turbulence properties from the single-phase simulation.

while the values in the table were determined by averaging over 10 independent realizations.

The volume- and time-averaged turbulent kinetic energy $K$ and turbulence intensity $u^{\prime}$ can be calculated either from the flow field or the energy spectrum $E(k)$ with

$$
K=\frac{3}{2} u^{\prime 2} \equiv \frac{1}{2} \bar{u} \cdot \boldsymbol{u} \equiv \int_{0}^{k_{\max }} E(k) \mathrm{d} k .
$$

Values, obtained from either the velocity field or the energy spectrum, are equal by definition.

The rate of energy dissipation can be determined directly using the rate of deformation tensor or, alternatively, can be calculated from the dissipation spectrum:

$$
\epsilon \equiv v \overline{\left(\frac{\partial u_{i}}{\partial x_{j}}+\frac{\partial u_{j}}{\partial x_{i}}\right) \frac{\partial u_{j}}{\partial x_{i}}} \equiv 2 v \int_{0}^{k_{\max }} k^{2} E(k) \mathrm{d} k .
$$

The gradients of the deformation rate tensor are inherently contained in the latticeBoltzmann scheme (Eggels \& Somers 1995). Using these gradients, no spatial differentiation of the velocity field is required to compute the rate of energy dissipation. The rate of energy dissipation computed this way is indicated in the table as $\epsilon_{l B}$, while the rate of energy dissipation calculated through the energy spectrum is indicated as $\epsilon_{E}$. The two values only differ by $1 \%$, indicating that the deformation rate contained in the lattice-Boltzmann scheme is consistent with the velocity field. The Kolmogorov length and time scales are computed using $\epsilon_{l B}$. The value of $\eta$ indicates that the input Kolmogorov length scale is recovered well. The minimum Kolmogorov length in the table is the smallest value encountered locally in instantaneous realizations of the flow field. This value can be regarded as a measure for the strongest gradients in the simulations. Since this value still satisfies the $k_{\max } \eta>1$ constraint, good quality of the DNS is ensured.

In isotropic turbulence simulations, the flow is often characterized using the Taylor microscale and the corresponding Reynolds number,

$$
\begin{aligned}
\lambda & \equiv\left(\frac{15 v u^{\prime 2}}{\epsilon}\right)^{1 / 2}, \\
R e_{\lambda} & \equiv \frac{u^{\prime} \lambda}{v} .
\end{aligned}
$$

$R e_{\lambda} \simeq 61$, as found in our simulation, has been simulated by various authors on grids of the order of $64^{3}$ to $96^{3}$ points (e.g. Eswaran \& Pope 1988; Overholt \& 


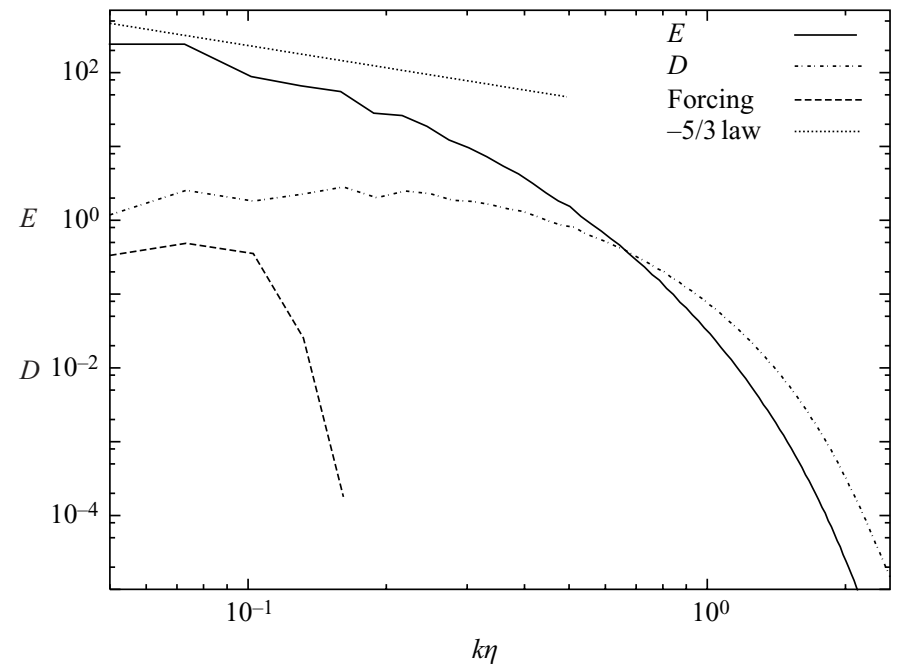

FiguRE 3. Scaled turbulent kinetic energy $\left(E=E(k) /\left(\epsilon^{2 / 3} \eta^{5 / 3}\right)\right)$ and dissipation $(D=$ $\left.2 v k^{2} E(k) /(\epsilon \eta)\right)$ spectra. 'Forcing' indicates the position of the forcing spectrum and is not to scale. $-5 / 3$ law indicates the spectral decay associated with the inertial subrange.

Pope 1998; Boivin et al. 1998; Reade \& Collins 2000) while Reynolds numbers of approximately 200 have been obtained on grids of $256^{3}$ points (Yeung \& Zhou 1997). The Taylor Reynolds number in our simulations remains relatively low with respect to the grid size. This is the direct consequence of our conservative choice of the Kolmogorov length of 1.2 grid units.

In figure 3 the normalized energy spectrum and the dissipation spectrum of the single-phase simulation are given. The figure contains the forcing spectrum to indicate its position in wavenumber space. The forcing dominates the turbulent motion at the largest scales, which is visible in the shape of the energy spectrum at the low wavenumbers. As a reference a $-5 / 3$ line is plotted in the figure. The $-5 / 3$ slope in the simulations is mainly found in the region where the forcing spectrum is present and due to the low Reynolds number of the simulation cannot be associated with the existence of a true inertial subrange. This is further demonstrated by the shape of the normalized dissipation spectrum, which indicates that dissipation takes place throughout the whole spectrum, but extends further into larger wavenumbers than the energy spectrum. The spectra are comparable to those presented by e.g. Eswaran \& Pope (1988), Sundaram \& Collins (1997) or Alvelius (1999).

Four different integral length scales of the turbulence are presented in table 2 . The integral length scale $\Lambda_{K}$ is calculated from the turbulence intensity and the rate of energy dissipation with the scaling law,

$$
\Lambda_{K}=\frac{u^{\prime 3}}{\epsilon} .
$$

A second integral length scale is computed from the energy spectrum:

$$
\Lambda_{E}=\frac{\pi}{2 u^{\prime 2}} \int_{0}^{k_{\max }} \frac{E(k)}{k} \mathrm{~d} k
$$

which is the length scale derived from the integration of the velocity correlation function. For reference, the longitudinal and transverse integral length scales $\Lambda_{f}$ 


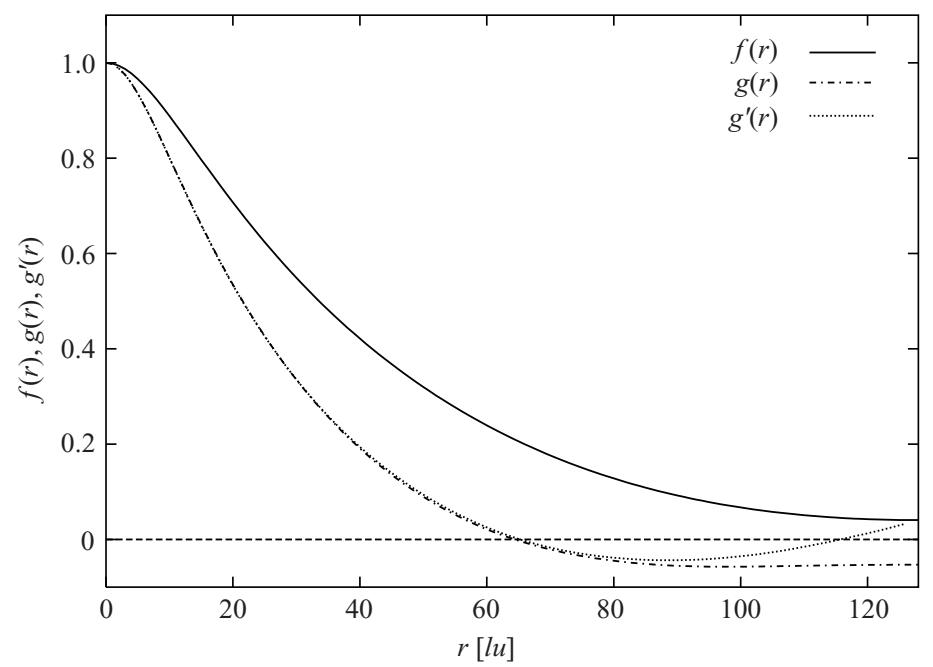

FIGURE 4. Longitudinal $f(r)$ and lateral $g(r)$ correlation functions of the turbulent simulation. $g^{\prime}(r)$ is the value of $g(r)$ as computed from $f(r)$ with relation (3.7).

and $\Lambda_{g}$, obtained from integration of the longitudinal and transverse velocity correlation functions $f(r)$ and $g(r)$ are also given. In isotropic turbulence their ratio is approximately 2 , which is the case here (Hinze 1975). Based on these length scales, integral time scales can be calculated, using $T_{i}=\Lambda_{i} / u^{\prime}$. The integral time scale $T_{K}$, calculated from the kinetic energy, is the eddy turnover time or eddy lifetime and is identical to $K / \epsilon$.

The transverse and lateral correlation functions $f(r)$ and $g(r)$, given in figure 4, show the typical behaviour for turbulent flow. They were found to be comparable to correlation functions obtained by Eswaran \& Pope (1988) and Overholt \& Pope (1998), who used both a different numerical method for solving the Navier-Stokes equations and a different forcing algorithm. A deviation from natural turbulent flows is that at larger separations the correlation functions do not reduce to zero. This is a result of the forcing scheme that is imposed on a periodic domain.

A relation between the longitudinal and lateral correlation functions $f(r)$ and $g(r)$ is given by (Hinze 1975, pp. 185),

$$
g(r)=f(r)+\frac{r}{2} \frac{\partial f(r)}{\partial r} .
$$

In figure 4, $g^{\prime}(r)$ denotes $g(r)$ based on $f(r)$ and equation (3.7) and shows excellent agreement with $g(r)$ at shorter separations. The deviation at larger separations may differ because the correlation functions do not drop to zero.

\section{Two-phase direct numerical simulations}

\subsection{Particle-phase definition}

Five simulations were performed (see table 3) where the volume fraction $\left(\Phi_{v}=V_{p} / V_{t o t}\right)$ was varied at a constant density ratio (simulations $S_{1}$ to $S_{3}$ ), and the particle density was varied at a constant volume fraction (simulations $S_{4}, S_{2}$ and $S_{5}$ in increasing order). In simulations $S_{4}, S_{2}$ and $S_{5}$, the particle densities were chosen such that the total mass of the system $\left(m_{t o t}=m_{p}+m_{f}\right.$, while $\left.\Phi_{m}=m_{p} / m_{f}\right)$ of $S_{4}$ corresponds to that of $S_{1}$ and $S_{5}$ to that of $S_{3}$. 


\begin{tabular}{lcccccc}
\hline \multirow{2}{*}{ Parameter } & \multicolumn{3}{c}{ Constant density ratio } & & \multicolumn{2}{c}{ Constant volume fraction } \\
\cline { 2 - 4 } & $S_{1}$ & $S_{2}$ & $S_{3}$ & & $S_{4}$ & $S_{5}$ \\
$N_{p}$ & 773 & 2200 & 3868 & & 2200 & 2200 \\
$\Phi_{v}$ & 0.020 & 0.057 & 0.100 & 0.057 & 0.057 \\
$\rho_{p} / \rho_{f}$ & 1.414 & 1.414 & 1.414 & & 1.146 & 1.728 \\
$\Phi_{m}$ & 0.029 & 0.085 & 0.157 & & 0.069 & 0.104 \\
$m_{t o t} \times 10^{6}$ & 16.92 & 17.17 & 17.47 & & 16.92 & 17.47 \\
$\tau_{p}$ & 1369 & 1369 & 1369 & & 1109 & 1673 \\
$S t_{K}$ & 0.207 & 0.207 & 0.207 & & 0.168 & 0.254 \\
$S t_{E}$ & 0.610 & 0.610 & 0.610 & & 0.494 & 0.745 \\
$S t_{\eta}$ & 4.845 & 4.845 & 4.845 & & 3.926 & 5.921
\end{tabular}

TABLE 3. Definition and properties of the particle phase for suspension simulations $S_{1}$ to $S_{5}$. The particle input radius was set to $4[l u]$ for all simulations, the corresponding hydrodynamic radius was $4.696[l u]$.

Particle inertia is characterized by the particle Stokes number, which for particles suspended in a turbulent flow field can be defined as the ratio of particle relaxation time $\left(\tau_{p}\right)$ and a relevant turbulent time scale,

$$
S t_{i}=\frac{\tau_{p}}{T_{i}}
$$

As particle relaxation time scale the Stokes relaxation time $\left(\tau_{p}=\rho_{p} d_{p}^{2} / 18 \mu\right)$ is used. This time scale is obtained from the equation of motion of a particle in the Stokes regime, i.e. where $R e_{p} \ll 1$. Although it is anticipated that the particle Reynolds number in our simulations will be $O(10)$ and nonlinear drag effects will be present, this particle relaxation time is used since it is a readily available time scale that is used in a large number of studies on turbulent particle-laden flows and therefore provides a basis for comparison (e.g. Sundaram \& Collins 1997; Boivin et al. 1998). For the turbulent time scale often the Kolmogorov time scale is chosen (see e.g. Sundaram \& Collins 1997; Sommerfeld 2001). This, however, is typically done for simulations where the particle size is much smaller than the Kolmogorov length scale. As table 2 already indicates, at least five different time scales can be determined for our simulations. With both a particle size that is in between Kolmogorov and macroscopic length scales and a relaxation time that is in between the Kolmogorov and integral time scales, it is not straightforward which time scale will dominate the particle motion and therefore is the most appropriate to insert in the expression for the Stokes number (4.1). Therefore, three different Stokes numbers are given in table 3. $S t_{K}$ and $S t_{E}$ are based on the corresponding integral time scales while $S t_{\eta}$ is based on the Kolmogorov time scale. The Stokes numbers indicate that the time scale of particle inertia is approximately 5 times larger than the Kolmogorov time scale and about half the integral time scale.

At the start of a simulation, the particles were placed randomly and without contact in a fully developed single-phase turbulent flow field. The initial flow field was identical for each simulation and the particles initially had zero velocity. As a result, the fluid flow field at the initial time step was clearly not consistent with the particle field. The particle velocity field had to adjust to the flow field and the flow field locally had to adjust to the presence of the particles before they were in agreement. This initial stage of development of the suspension flow field required a 


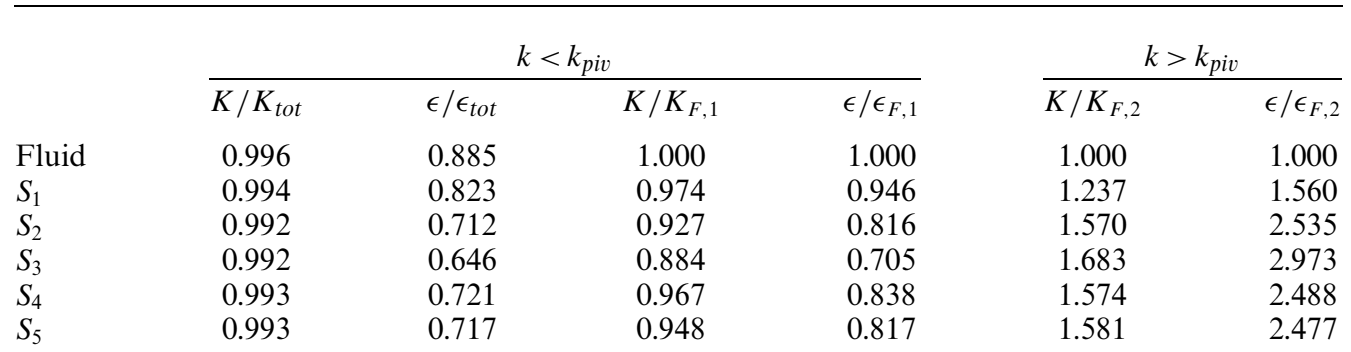

TABLE 4. Relative distribution of kinetic energy and rate of energy dissipation over wavenumbers smaller or larger than $k_{p i v} . K / K_{\text {tot }}$ and $\epsilon / \epsilon_{\text {tot }}$ indicate the kinetic energy and energy dissipation in the spectrum at wavenumbers larger than $k_{p i v}$, relative to the total kinetic energy in that simulation. $K / K_{F, i}$ and $\epsilon / \epsilon_{F, i}$ present the change in kinetic energy and rate of energy dissipation, relative to kinetic energy or energy dissipation contained in the single-phase simulation at the interval smaller or larger than $k_{p i v}$.

few hundred time steps (typically faster than the integral time of the turbulent flow field or the relaxation time scale of the particles). After the initial few hunderd time steps, the particle kinetic energy had developed to a statistically steady state. This initial stage was not considered in further analysis of the suspension data.

The order in which the precalculated force fields were imposed was identical for all simulations, in order to have identical time series of the forcing signal. Obviously, the evolution of the flow fields differed per case due to changes in the flow field that originate from the presence of the particle phase. The motion of the particles modifies the flow field directly, which furthermore results in a change of the uncontrolled contribution $P_{2}$ (see also $\S 2.2$ ).

\subsection{Kinetic energy distribution in the turbulent suspension simulations}

In figure $5(a-d)$, cross-sections of the flow field are given at increasing particle concentration. In the single-phase simulation of figure 5(a), a number of vortical structures can be seen, typical of a turbulent flow field. At increasing particle concentration, the pattern of the rate of energy dissipation is altered. An increase of the rate of energy dissipation close to the particle surfaces can be observed in these figures as well as in the close-up image of figure 5(e).

The presence of particles changes the distribution of kinetic energy and rate of energy dissipation over the different length scales, as can be seen in the spectra of figure 6 . This change in the energy spectrum is quantified in table 4. The spectra were determined by averaging 10 realizations of the full fluid domain and contain the contribution of all grid nodes, both from the bulk fluid domain as well as from the inside of the particles.

At the largest scales, the fluid motion is dominated by the turbulent forcing. As a result, little difference exists between the spectra of the various simulations for $k / k_{d} \leqslant 0.1$. At larger wavenumbers, the fluid spectrum is influenced less by the forcing and the impact of the particles on the kinetic energy can be clearly distinguished. Figure 6(a) shows that an increase in particle concentration reduces the fluid kinetic energy at intermediate wavenumbers $\left(0.15<k / k_{d}<0.7\right)$. At larger wavenumbers the spectra cross at a clear pivot point at wavenumber $k_{p i v}\left(k_{p i v} / k_{d} \simeq 0.72\right)$. It is striking that this pivot point is found at the same wavenumber for all simulations. This is probably due to the fact that the length scales in the simulations remained practically constant. The turbulent Reynolds number was the same throughout the simulations, 

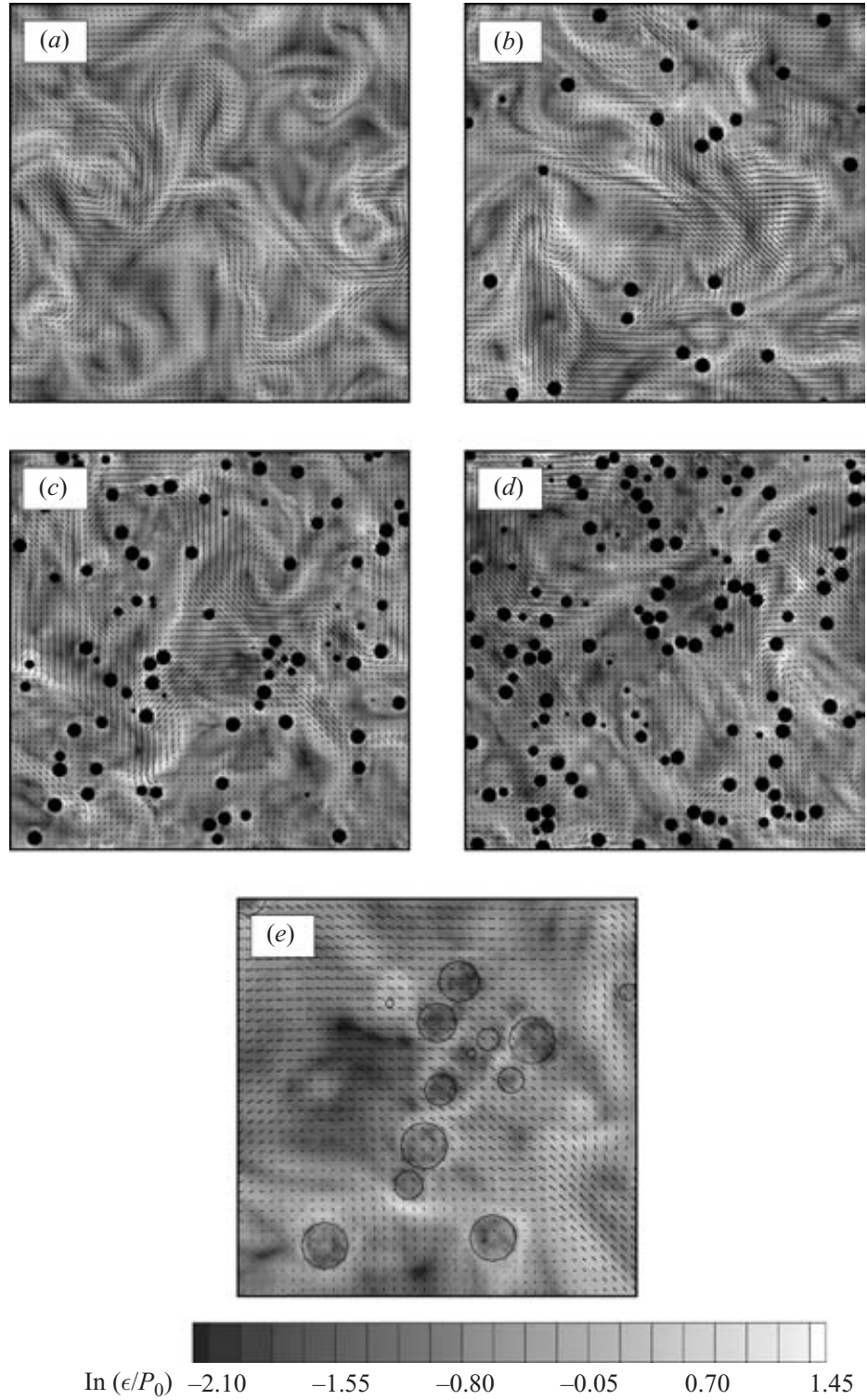

FigURE 5. Cross-sections of single realizations of the flow field of the single-phase simulation, (b) $S_{1},(c) S_{2}$ and $(d) S_{3}$ and (e) a close-up of the centre of the flow field of $S_{2}$. The particles are plotted at their position, intersected by the plane of view. In $(e)$, circles are plotted at the cross-section of the particles with the flow field to show the flow field on the inside of the particles. The shading indicates the logarithmic value of the rate of energy dissipation. The vectors represent the velocity in the fluid and have been plotted at a linear interval of 1 out of 4 for $(a-d)$ and 1 out of 2 for $(e)$.

which indicates that the ratio of the macroscopic to microscopic turbulent length scales in the simulation remained the same, while the particle size was also constant throughout the simulations.

The kinetic energy spectrum in figure 6 exhibits a smooth behaviour up to wavenumbers around $k / k_{d}=1.5$. At larger wavenumbers $\left(k / k_{d}>1.5\right)$ the energy 

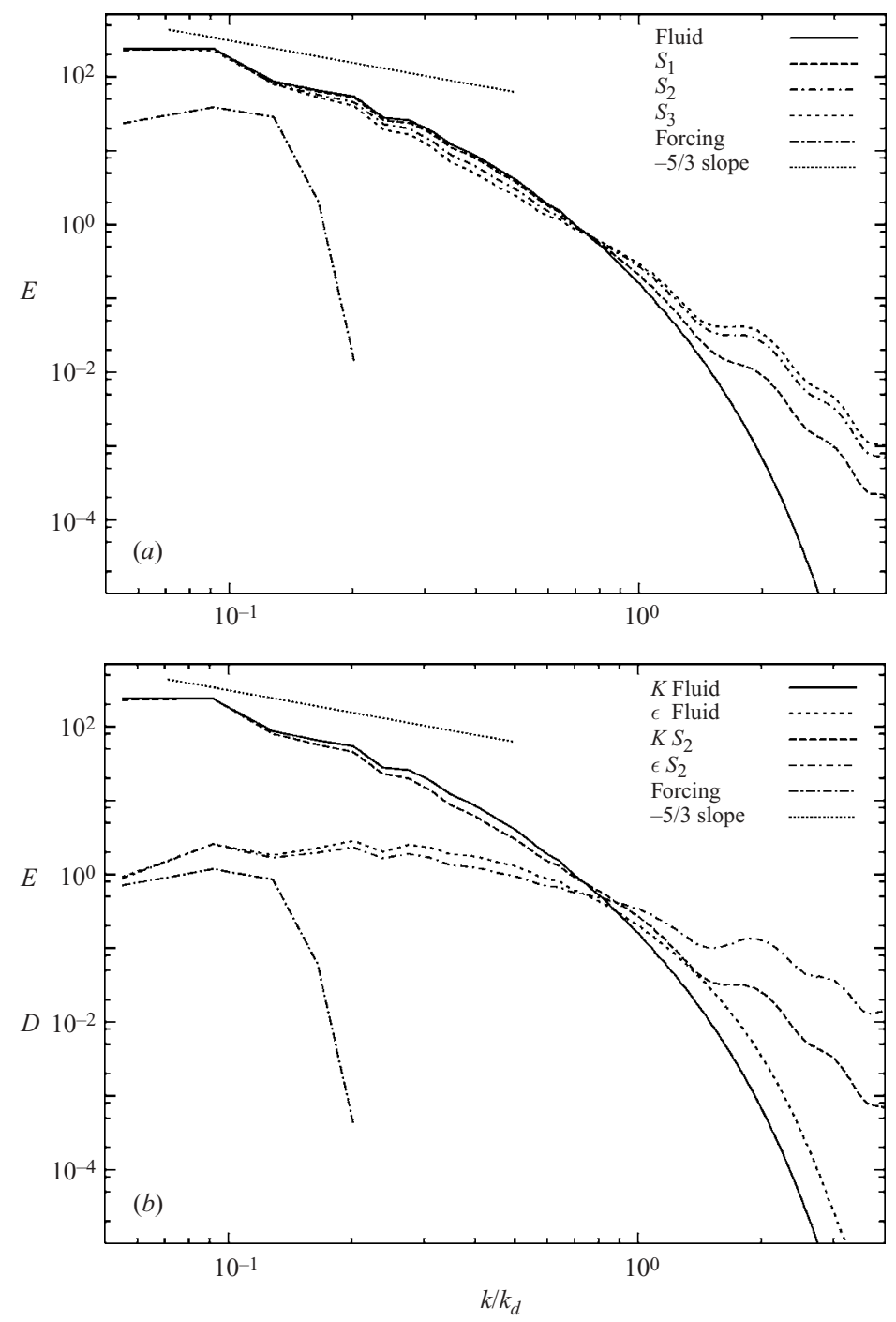

FIGURE 6. (a) Scaled energy spectra $\left(E=E(k) /\left(\epsilon^{2 / 3} \eta^{5 / 3}\right)\right)$ of the single-phase simulations ('Fluid') and simulations $S_{1}$ to $S_{3}$ and $(b)$ energy and dissipation spectra $\left(D=2 v k^{2} E(k) /(\epsilon \eta)\right)$ of single-phase simulation and suspension simulation $S_{2}$. The forcing spectrum and $-5 / 3$ slope are included (see also figure 3 ). The wavenumber axis is scaled with the particle diameter wavenumber $k_{d}=2 \pi / d_{p}$.

spectrum shows a fluctuating behaviour due to the presence of the solid surface of the particles. The column of table 4 that presents $K / K_{\text {tot }}$ shows that these fluctuations only make a small contribution to the total kinetic energy of the system, since more than $99 \%$ of the kinetic energy is contained at wavenumbers larger than the pivot wavenumber.

At the same time, the dissipation spectrum as well as $\epsilon / \epsilon_{\text {tot }}$ show that this fluctuating behaviour of the energy spectrum increases energy dissipation at the smallest scales significantly. The rate of energy dissipation is strongly redistributed by the action of the particles. A decrease from $89 \%$ for single phase to $65 \%$ for $S_{3}$ is observed. This increase in dissipation is a result of the discrete particle surfaces that generate 
velocity gradients in the fluid close to the particle surface exterior as well as interior. The contour plot of figure 5(e) indeed shows an increase in energy dissipation at the surface of the particles while in the interior of the particles the rate of energy dissipation is decreased.

From the flow field the ratio of energy dissipation on the inside of the particles to dissipation in the overall domain was computed. It was found that on average the rate of energy dissipation inside the particles was approximately 0.7 times the energy dissipation as averaged over the whole domain. This confirms that the flow field inside the particles generally is more quiescent. As a result, the fluid inside of the particles makes a contribution to the energy dissipation spectrum that is much smaller than the change of the dissipation spectrum indicated by $\epsilon / \epsilon_{\text {tot }}$ in table 4 .

From the flow field the rate of energy dissipation in a layer of cells of two lattice units around the surface of the spheres was also computed. In this layer the rate of energy dissipation was found to be approximately 1.5 times the mean dissipation, which clearly confirms the image that the dissipation is strongly increased near the particle surfaces. Furthermore, from this increased rate of energy dissipation a Kolmogorov length near the surface of the particles was estimated to be approximately 1.1 $[l u]$. This indicates a proper resolution for the flow field near the particles.

Table 4 demonstrates that changing the particle volume fraction reduces the kinetic energy of the flow field much more than changing the particle inertia. By increasing the number of particles, the total amount of particle surface is increased, which results in an increase of the rate of energy dissipation. Increasing the particle inertia will reduce the response of particles to the turbulent flow field (see e.g. Abrahamson 1975; Kruis \& Kusters 1997). This will increase the slip velocity of the particles, thus also enhancing the velocity gradients near the surface of the particles, but this effect is apparently less strong. $K / K_{F, 1}$ in table 4 demonstrates that the kinetic energy in simulation $S_{5}$ is reduced by approximately $5 \%$ while for case $S_{3}$ this is roughly $22 \%$.

Modulation of the kinetic energy spectrum by the presence of particles has been studied via direct numerical simulations by a number of authors (e.g. particles in forced isotropic turbulence by Squires \& Eaton (1990) and Boivin et al. (1998) and in decaying isotropic turbulence by Elghobashi \& Truesdell (1993)). In the work of these authors, the particles were very much smaller than the numerical grid, the volume fraction was on the order of $10^{-4}$, while the mass loading was considerable $\left(\Phi_{m}\right.$ up to 1.0). The particle inertia was characterized by the particle relaxation time while Stokes drag was assumed to dominate the particle motion. In these two-way coupled simulations the interaction between the particles and the fluid phase was imposed by projecting the force that acts on the particles back to the fluid grid.

Clearly, large differences exist between this approach and our simulations. In our simulations all scales of the fluid motion are resolved, whereas details of the flow field near the particle are not captured in the work presented by the authors mentioned above. Still, for a mass loading of approximately $10 \%$, the work of these authors indicates a decrease in turbulent kinetic energy of approximately $10 \%$, which is comparable to the decrease observed in simulations $S_{2}$ and $S_{3}$ in table 4.

In the work of Boivin et al. (1998), simulations are presented where both the particle mass loading and the particle Stokes numbers are in the same range as in our simulations. In that work, a decrease of the rate of energy dissipation in the fluid phase of approximately $20 \%$ was observed. This decrease of energy dissipation is caused by the particle drag, given by the Stokes drag relation, which works as an energy sink for the unresolved part of the flow field at the particle scale. In our simulations a practically constant power input is warranted and because all details of the flow 
field are captured an equilibrium between power input and dissipation will exist. The total energy dissipation in the fluid phase is therefore practically constant. On the other hand, our simulations indicate a decrease of the rate of energy dissipation at large scales (table $4, k<k_{p i v}$ ) by $5 \%$ to $30 \%$, which is of a comparable order to that found by the above authors. This demonstrates that, although the simulation approach of the above-mentioned authors lacks the detail of the small-scale fluid motion as present in our simulations, the large-scale phenomena of both simulation approaches are consistent.

\section{Analysis of particle-phase behaviour}

In this section, the motion of the particle phase in our simulations is studied in detail to characterize the particle collision behaviour observed in the simulations and to characterize the relation between the particle motion and the turbulent flow field. As stated in the introduction, particle collisions in turbulent suspensions have been studied by a large number of authors and a considerable theoretical framework exists to describe and model particle motion in turbulent flow fields. In the following paragraphs we will first give a short review of the collision theory as presented by Wang et al. (2000). This theoretical basis will subsequently be used to discuss characteristic features of our suspension simulations such as the distribution of relative particle velocities, local particle accumulation and particle collisions.

\subsection{A short review on the formulation of the collision kernel}

Two limiting cases of turbulent suspension flows are dilute flows, where the particle motion is dominated by hydrodynamic transport effects, and dense flows, where the motion of the particles is dominated by inter-particle collisions (Sommerfeld 2001). Based on their high volume fraction one would expect that the suspensions studied here are well in the dense regime. However, the two regimes are not only characterized by the volume fraction. The ratio of the particle relaxation time and the average time between two collisions $\left(\tau_{c}\right)$ gives a more precise indication of the regime of the suspension. In the dilute flow limit $\tau_{p} \ll \tau_{c}$; in the dense flow limit $\tau_{p} \gg \tau_{c}$. In dense two-phase flows, particles are not able to respond to the fluid flow between successive collisions. This applies to heavy particles in low volume concentrations or to light particles at higher volume concentrations.

We are interested in the behaviour of turbulent slurries at moderate to high volume fractions, that consist of large particles $\left(d_{p}>\eta\right)$. The average time between particle collisions is $\tau_{c}=1 / f_{c}$, the inverse of the collision frequency $f_{c}$. For a monodisperse system of $N_{p}$ particles in a volume $\Omega$, the collision frequency is given by

$$
f_{c}=\frac{1}{\tau_{c}}=\frac{\dot{\mathscr{N}}_{c}}{n_{0}}=\frac{n_{0}}{2} \Gamma
$$

where $\dot{\mathscr{N}}_{c}$ is the rate of collisions per unit volume, $n_{0}=N_{p} / \Omega$ is the particle number concentration. In equation (5.1) the average collision kernel $\Gamma$ is defined. In order to estimate the regime we are in, an upper bound estimate for $\Gamma$ is (Abrahamson 1975)

$$
\Gamma=\sqrt{\frac{16 \pi}{3}} R^{2} u_{p}^{\prime}
$$

where $u_{p}^{\prime}$ is the particle r.m.s. velocity and $R=d_{p}$. Based on this approximation the ratio $\tau_{p} / \tau_{c}$ ranges from 0.2 to 0.8 in our simulations. As a result, collisions as well as hydrodynamic forces will contribute to the overall motion of the particles. 
An expression for the collision kernel $\Gamma$ can be derived based on two different approaches, known as the cylindrical and the spherical approaches. Wang et al. (1998) give an analysis of both formulations and demonstrate that the spherical formulation is the more appropriate way to describe the collision process. We will restrict ourselves to the spherical formulation. According to the original concept of Saffman \& Turner (1956), a collision sphere is defined as a central sphere with a radius $R=r_{i}+r_{j}$ which for monodisperse systems is $R=2 r_{p}$. In the centre of this collision sphere, a particle is placed. The collision kernel is given by the radial flux of particles through the surface of the collision sphere, given by the mean inward velocity $w_{r}^{-}$times the probability $P$ of observing this velocity,

$$
\Gamma_{c}=4 \pi R^{2} P w_{r}^{-}
$$

where $w_{r}$ is the radial component of the relative velocity $\mathbf{w}_{i j}=\boldsymbol{u}_{p, i}-\boldsymbol{u}_{p, j}$ and $P$ is the probability of observing $w_{r}<0$. If radial influx and outflux are in equilibrium, the collision kernel can be rewritten in terms of the absolute mean radial velocity $\left\langle\left|w_{r}\right|\right\rangle$, which is given by

$$
\left\langle\left|w_{r}\right|\right\rangle=\int_{-\infty}^{0}-w P(w) \mathrm{d} w+\int_{0}^{\infty} w P(w) \mathrm{d} w=P w_{r}^{-}+(1-P) w_{r}^{+}
$$

where the angle brackets denote ensemble averaging over all directions in space and time averaging. The ratio of net inward to net outward flux is given by

$$
C_{p} \equiv \frac{P w_{r}^{-}}{(1-P) w_{r}^{+}}
$$

which should be equal to 1 for a system in equilibrium. Wang et al. (2000) indicate that the ratio $w_{r}^{-} / w_{r}^{+}$can be viewed as a rough measure for the compressibility of the particle velocity field. Both $C_{p}$ and $w_{r}^{-} / w_{r}^{+}$will be discussed for our simulations in a later section. When substituting equations (5.4) and (5.5) into (5.3), assuming equilibrium between in- and outflux, the spherical formulation is obtained:

$$
\Gamma^{s p h}=2 \pi R^{2}\left\langle\left|w_{r}\right|\right\rangle \text {. }
$$

If one assumes the particle velocity to be Gaussian distributed, then the relative velocity magnitude $\left\langle\left|w_{r}\right|\right\rangle$ can be related to the variance of the distribution as $\left\langle\left|w_{r}\right|\right\rangle=\sqrt{2 / \pi} \sigma_{w}$ with $\sigma_{w}$ given by

$$
\sigma_{w}=\sqrt{w_{r}^{2}}=\left[2\left(u_{p}^{\prime}\right)^{2}-2\left\langle u_{r, 1} u_{r, 2}\right\rangle\right]^{0.5}
$$

and $u_{r, i}$ the radial velocity component of particle $i$. The relative velocity magnitude of the particles therefore is a function of the velocity correlation of the particles at contact. For isotropic turbulence, the correlation $\left\langle u_{r, 1} u_{r, 2}\right\rangle$ of the radial velocity of the fluid is given by the longitudinal velocity correlation function $f(r)$. An equivalent expression for the particle transverse relative velocity is given by

$$
\sigma_{w}=\sqrt{w_{t}^{2}}=\left[2\left(u_{p}^{\prime}\right)^{2}-2\left\langle u_{t, 1} u_{t, 2}\right\rangle\right]^{0.5}
$$

where $\left\langle u_{t, 1} u_{t, 2}\right\rangle$ can be compared with the fluid lateral correlation function $g(r)$.

The particle properties and particle-turbulence interaction simultaneously determine the particle correlation function which in general deviates from the fluid correlation functions. The effect of the particle size on the collision rate is demonstrated by evaluating the longitudinal correlation function $f(r)$ at contact. In our simulations, the separation $R$ of the centres of mass of two particles at contact is about 8 times the Kolmogorov length. At this separation, the fluid correlation functions $f(r)$ and 
$g(r)$ have already dropped considerably. If one assumes that the particle velocity correlation is equal to $f(r)$, then $\sqrt{w_{r}^{2}}$ is given by

$$
\sqrt{w_{r}^{2}}=\left[2\left(u_{p}^{\prime}\right)^{2}(1-f(R))\right]^{0.5} .
$$

Since $f(R)<1$, the relative velocity is larger at contact as $R$ becomes larger and, as a result, an increase in particle size increases the collision rate. In the following section, we will discuss the lateral and transverse relative velocities of the particles as a function of their separation.

The formulation of equation (5.6) holds for particles that exhibit a homogeneous, random spatial distribution. Finite-inertia particles in turbulent flows, however, have a tendency to collect in regions with low vorticity and high strain rate. As demonstrated by Sundaram \& Collins (1997), the impact of this 'preferential concentration' effect can be separated from the turbulent transport effect via the radial distribution function at contact, $g_{r}(R)$. By including this term, the spherical formulation of the collision kernel becomes

$$
\Gamma^{s p h}=2 \pi R^{2}\left\langle\left|w_{r}\right|\right\rangle g_{r}(R) .
$$

The radial distribution function $g_{r}(r)$ will be discussed to demonstrate the presence of particle accumulation and preferential concentration in our suspension simulations.

\subsection{Fluid and particle relative velocities}

Radial and transverse relative velocity components of the fluid single-phase simulation have been plotted in figure 7. The relative velocities in these plots are determined from $w_{i}=u_{i}(x+r)-u_{i}(x)$, where the separation distance $r$ had values of 1,8 and 32 lattice units. At $r=1$, the radial relative velocity reduces to the velocity gradient $\partial u / \partial x$ while the transverse relative velocity reduces to $\partial u / \partial y$. The figure demonstrates clearly how the distribution of the relative velocity changes with distance. As a reference, a normalized exponential and Gaussian distribution are given.

At a separation of 1 , we see that the radial relative velocity distribution is negatively skewed with a skewness value of -0.5 . This skewness is a typical characteristic of isotropic turbulence, also reported by e.g. Alvelius (1999) and Wang et al. (2000). It is further noted that, for a separation of $r=1$, the tail of the distribution follows the exponential curve at negative relative velocities, while at positive values the distribution has a Gaussian shape.

As the separation increases to $8[l u]$, the skewness decreases and the shape of the distribution tends to the Gaussian curve. When the separation further increases to $32[l u]$, the correlation decreases (see figure 4 ) and the distribution of the radial relative velocity becomes Gaussian. The relative velocity magnitude and the velocity correlation values corresponding to the data in figure 7 are given in table 5. The tendency of the relative velocities towards a Gaussian distribution is expected since the relative velocity of two uncorrelated Gaussian-distributed velocity components is again Gaussian distributed (see equations (5.7) and (5.8)).

The transverse relative velocity is also given in figure 7 . In an isotropic flow field no directional distinction can be made regarding the velocities in the plane perpendicular to the radial direction. The sign of the transverse relative velocity indicates velocities relative to an arbitrarily chosen coordinate system, which in this case corresponds to the coordinate system of the computational grid. The distribution must be symmetric by definition. The distribution of the transverse relative velocities exhibits an exponential shape at short separation distances and changes into a Gaussian distribution at larger separations. The Gaussian distribution 

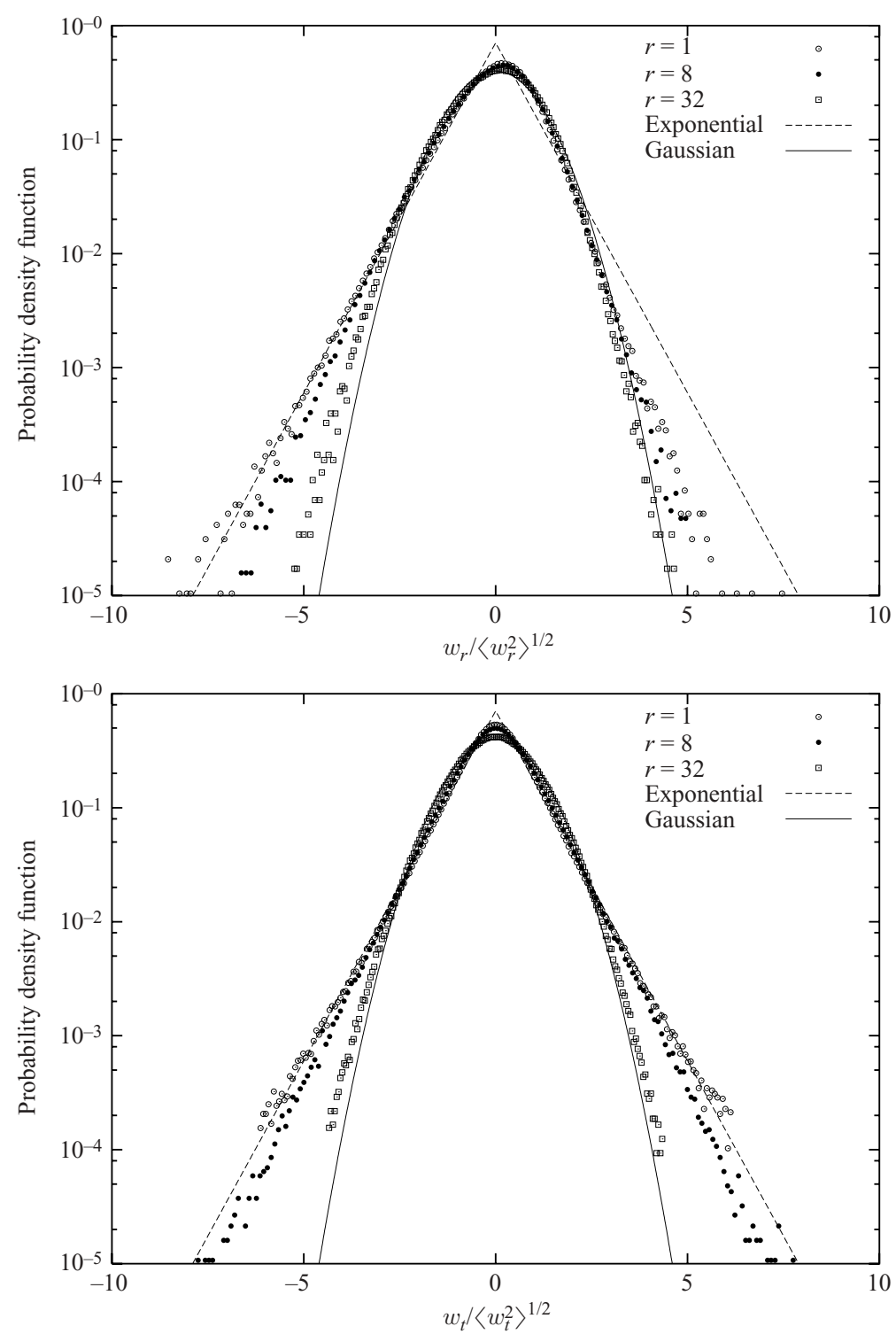

FiguRE 7. Distributions of the radial and transverse relative velocity components of the single-phase simulation. The relative velocities were determined at separation distances $r=1$, $r=8$ and $r=32[l u]$.

can be anticipated based on the same argument as given for the radial relative velocities (see equation (5.8)).

In table 5, the standard deviation or r.m.s. value of the radial and transverse relative velocity at the three separation distances is presented. Transverse r.m.s. velocities are larger than the radial components because $g(r)$ has a stronger decay with $r$ than $f(r)$.

For the computation of the radial and transverse velocity distributions of the particle phase, the radial and transverse velocity component need to be defined. The radial velocity is defined by $w_{r}=\boldsymbol{w} \cdot \boldsymbol{R} / \tilde{R}$, where $\boldsymbol{R}$ is the vector with length $\tilde{R}$, connecting the centres of any two particles. A definition for the transverse 


\begin{tabular}{|c|c|c|c|c|c|c|}
\hline & \multicolumn{3}{|c|}{ Fluid } & \multicolumn{3}{|c|}{$S_{2}$} \\
\hline & $r$ & $\left(w_{i}^{2}\right)^{1 / 2} \times 10^{3}$ & $\left\langle u_{i, 1} u_{i, 2}\right\rangle / u^{\prime 2}$ & $s$ & $\left(w_{i}^{2}\right)^{1 / 2} \times 10^{3}$ & $\left\langle u_{i p, 1} u_{i p, 2}\right\rangle / u_{p}^{\prime 2}$ \\
\hline \multirow[t]{3}{*}{ Radial } & 1 & 0.91 & 0.998 & 0.5 & 3.12 & 0.979 \\
\hline & 8 & 6.63 & 0.918 & 2.5 & 6.90 & 0.895 \\
\hline & 32 & 16.49 & 0.494 & 31.5 & 16.20 & 0.423 \\
\hline \multirow[t]{3}{*}{ Transverse } & 1 & 1.28 & 0.997 & 0.5 & 7.20 & 0.886 \\
\hline & 8 & 8.98 & 0.850 & 2.5 & 9.17 & 0.815 \\
\hline & 32 & 19.91 & 0.262 & 31.5 & 19.26 & 0.184 \\
\hline
\end{tabular}

TABLE 5. Standard deviation of the relative velocities of the fluid for the single-phase simulation and for the particle phase in simulation $S_{2}$, both determined at three different separation distances $r$ or $s$.

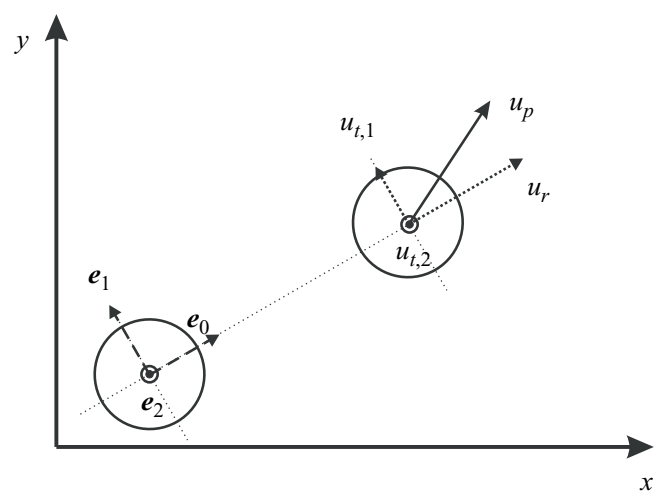

FIGURE 8. Two-dimensional representation of the axial and transverse vector decomposition for a particle pair.

component is given by $w_{t}=|\boldsymbol{w} \times \boldsymbol{R}| /|\tilde{R}|$ (Wang et al. 2000). The transverse velocity vectors, projected in the plane perpendicular to $\boldsymbol{R}$, are not necessarily aligned. In our simulations we therefore chose to decompose the two vectors into two orthogonal components in the plane perpendicular to the connecting axis using the coordinate system defined by Alvelius (1999). The principal axis (see figure 8) is given by $\mathbf{e}_{0}=\boldsymbol{R} /|\tilde{R}|$, while two axes orthogonal to $\mathbf{e}_{0}$ and to each other are given by the unit vectors $\mathbf{e}_{1}$ and $\mathbf{e}_{2}$,

$$
\begin{gathered}
e_{1, x}=\frac{e_{0, y}}{\left(e_{0, x}^{2}+e_{0, y}^{2}\right)^{1 / 2}}, \quad e_{1, y}=-\frac{e_{0, x}}{\left(e_{0, x}^{2}+e_{0, y}^{2}\right)^{1 / 2}}, \quad e_{1, z}=0, \\
e_{2, x}=\frac{e_{0, x} e_{0, z}}{e_{0}\left(e_{0, x}^{2}+e_{0, y}^{2}\right)^{1 / 2}}, \quad e_{2, y}=\frac{e_{0, y} e_{0, z}}{e_{0}\left(e_{0, x}^{2}+e_{0, y}^{2}\right)^{1 / 2}}, \quad e_{2, z}=-\frac{\left(e_{0, x}^{2}+e_{0, y}^{2}\right)^{1 / 2}}{e_{0}} .
\end{gathered}
$$

By using this coordinate system, the particle velocities can be decomposed in a longitudinal and two parallel velocities from which one radial and two transverse relative velocity components can be determined. The relative velocity distributions are now evaluated based on a coordinate system that changes direction depending on the particle pair that is evaluated. As a result of the isotropic flow field, characteristic statistical quantities such as the relative velocity distribution only depend on the separation $r$ and not on the direction of this separation. 

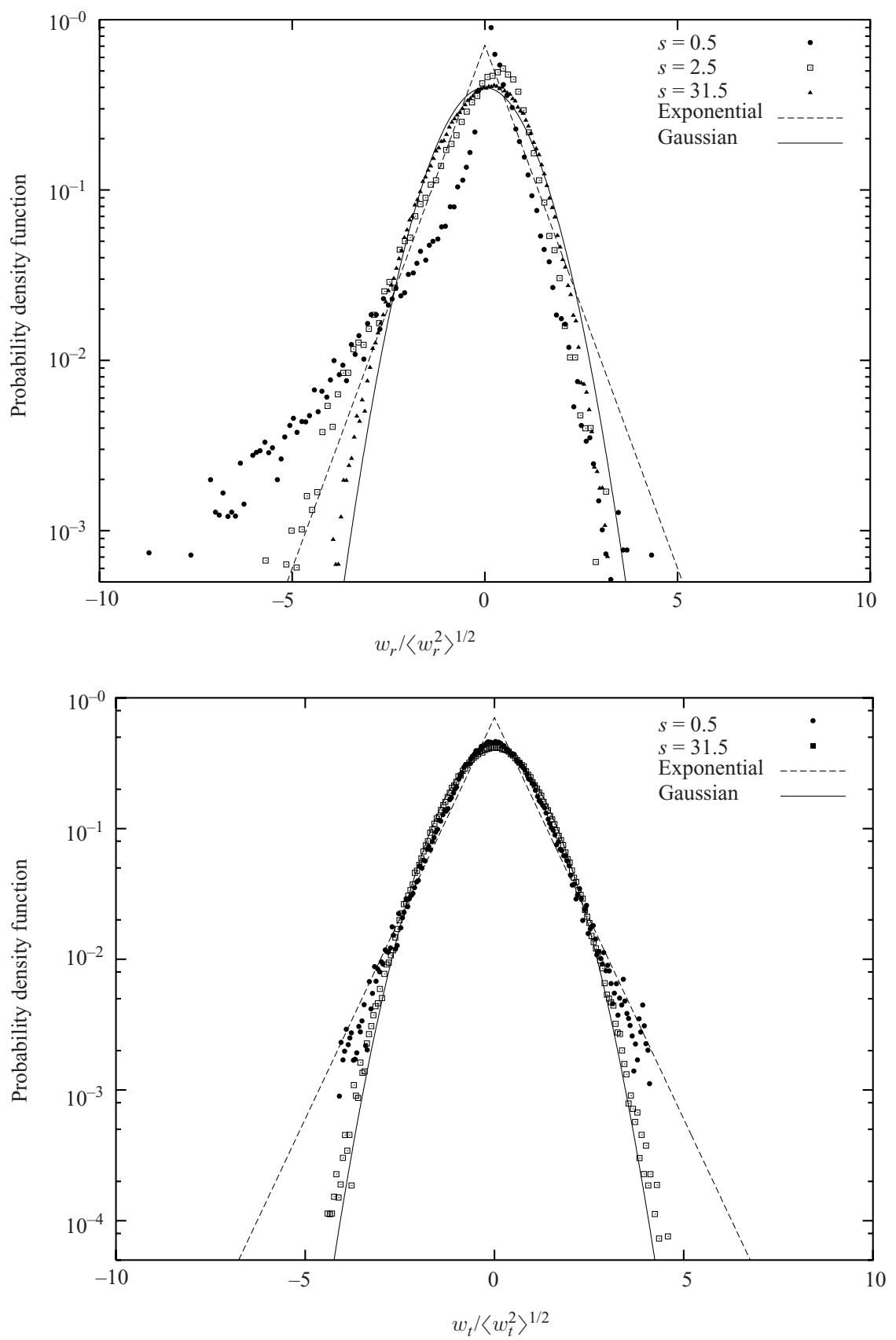

FIGURE 9. Distribution of the radial and transverse relative velocity components of simulation $S_{2}$. The relative velocities were determined from particles that were separated by $s=0.5 \pm 0.5$, $s=2.5 \pm 0.5$ and $s=31.5 \pm 0.5[l u]$, where $s$ indicates the separation gap between two spheres.

In figure 9 the relative velocity distributions of the particle phase of simulation $S_{2}$ are given at three different separations. The separation $s$ refers to the gap between the two sphere surfaces, i.e. $s=r-|R|$. For each realization statistical data were obtained from all $(1 / 2) N_{p}\left(N_{p}-1\right)$ particle pairs. The velocity distribution at a given separation 
$s$ was determined from all particles in the flow field that were separated by $s \pm 0.5[l u]$, and data were obtained over 75 realizations of the particle field at a time spacing of 470 time steps.

The distribution of the radial relative velocity component at $s=0.5[l u]$ is strongly asymmetric and is much more negatively skewed $(-2.43)$ than the distribution of the fluid velocity at a separation of $1[l u]$ (see figure 7 ). The negative relative velocity (i.e. approach velocity) contains a strongly stretched tail, while the positive relative velocity (separation) is found to lie practically inside the Gaussian distribution. As the separation increases, the skewness reduces and the tail in the distribution disappears. At $s=2.5$, the skewness has become -0.94 .

Strong negative skewness and stretched tails in the distribution are also observed in the results of Wang et al. (2000), who studied collision characteristics of small particles $\left(d_{p}<\eta\right)$ in one-way coupled simulations carried out in frozen flow fields. As the particle relaxation time in their simulations was increased from $\tau_{p} / \tau_{k}=0$ to $\tau_{p} / \tau_{k}=1$, the skewness in the distribution decreased to a minimum of -2.03 . In their results tails were observed in both the positive and the negative directions of the radial relative velocity distributions.

In our simulations, the distribution of the radial relative velocity reflects the behaviour of particles near contact, whose motion is strongly influenced by shortrange hydrodynamic particle-particle interactions. As two particles approach, the relative velocity of the particles decreases due to the dissipative action of the lubrication force and due to correlation of the velocity in the turbulent flow field.

The shape of the distribution at negative values is caused by two separate effects: (i) the tail in the distribution is a result of the velocities of particles that were initially separated by larger distances and had uncorrelated and hence larger relative velocities; (ii) the distribution contains contributions of particle pairs that were already in each other's vicinity and remain there, increasing the presence of low relative velocities in the p.d.f.

For two separating particles in close proximity (i.e. for positive radial velocities), the lubrication force becomes attractive, which suppresses the separating velocities and tends to keep particle pairs together. As a result, either sufficient inertia or the action of the turbulent flow field is required to separate particle pairs.

At higher values of $s$, the shape of the distribution changes rapidly. When $s$ increases from 0.5 to 2.5 , the short-range hydrodynamic interactions decay strongly, which results in a change of the p.d.f. towards a Gaussian distribution. At further separation, the distribution becomes practically Gaussian in a similar way to the distribution of the radial fluid velocity.

The transverse relative velocity distribution at closest separation shows a practically Gaussian distribution. This shape may be caused by both the particle inertia and the particle size. Due to their inertia, the motion of two particles near contact will be partially decorrelated. Further, since two particles near contact span approximately 20 lattice units, the transverse velocity correlation of the fluid that influences the particle motion has significantly reduced to approximately 0.6 over this distance. The fluid transverse relative velocity at this separation has become Gaussian distributed, leading to a Gaussian shape of the distribution of the transverse part of the particle relative velocity.

Table 5 shows that a large difference exists between the radial and the transverse relative velocity magnitude in the particle phase. The transverse velocity reduces by $20 \%$ as the separation reduces from 2.5 to $0.5[l u]$, while the radial velocity decreases by $55 \%$. This difference arises because in the radial direction the particle motion is 


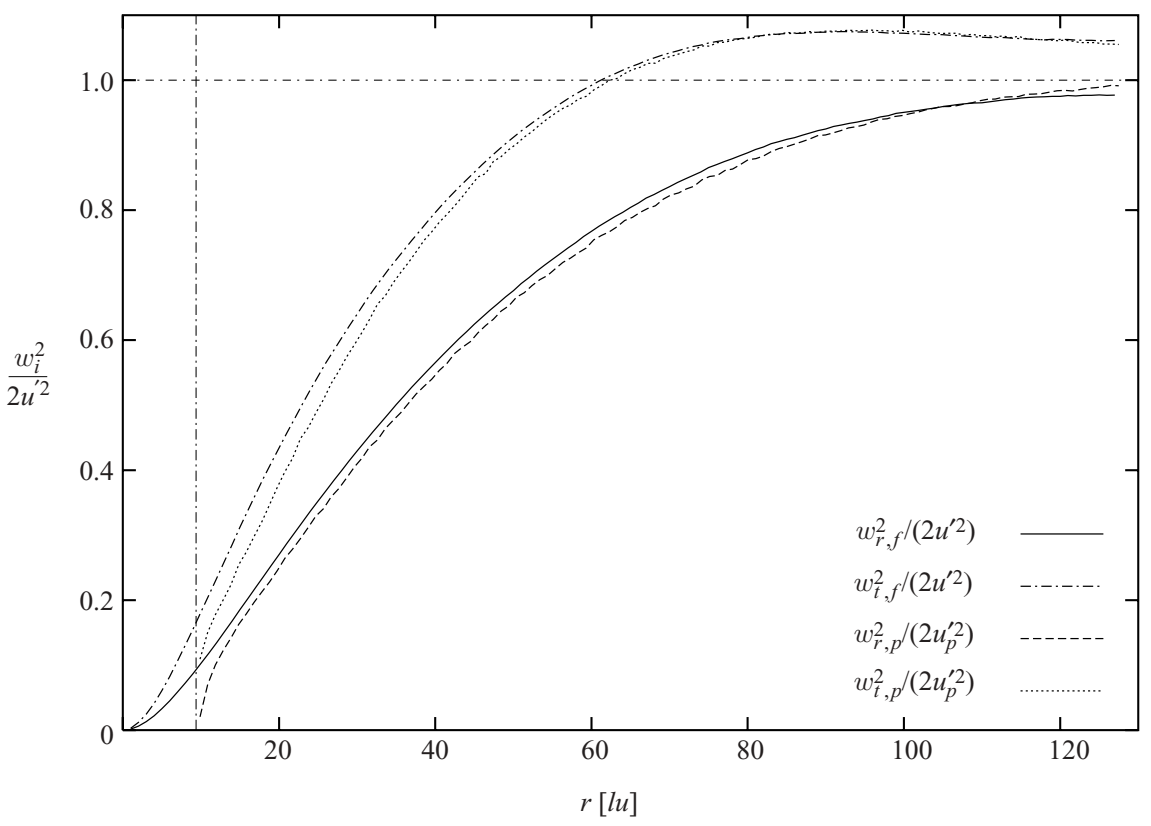

FIGURE 10. Normalized variance of radial and transverse relative velocity $\left(w_{i}^{2} /\left(2 u_{i}^{\prime 2}\right)\right)$ of the fluid and particle phase of simulation $S_{2}$, as a function of separation $r$. The vertical line indicates the separation $R$ at which there is contact between two particles.

strongly influenced by lubrication force while in the transverse direction only shear forces are active that are orders of magnitude smaller and consequently have a much smaller effect.

The normalized velocity variance of the radial and the transverse velocity components for the fluid and the particle phase are given in figure 10. For the fluid phase one can recognize the shape of the curves as $1-f(r)$ and $1-g(r)$, the longitudinal and transverse correlation functions (see also figure 4 and equations (5.7) and (5.8)). The particle relative velocity variance at larger separations follows but remains below that of the fluid phase, while towards small separations the values decay faster. The strong decay for particles close to contact is probably a result of the strong hydrodynamic interactions between particle pairs near contact due to lubrication forces. At close separations the particles interact with either repulsive or attractive forces, depending on whether particles approach or separate. As a result, the particle motion becomes more correlated, affecting both the radial and transverse velocity variance of the particles.

The flux-balance coefficient $C_{p}$ of equation (5.5) is given in figure $11(a)$ as a function of the separation $r$. It demonstrates that throughout the domain, the flux balance assumption is valid for both the fluid phase and the particle phase and the particle flux in the simulations has reached steady state.

The ratio $w_{r}^{-} / w_{r}^{+}$of figure 11(b) exhibits a monotonic increase at decreasing separation. According to Wang et al. (2000) this ratio is a measure of the compressibility of the (particle) velocity field. However, when $C_{p}$ equals 1 , as in our simulations, $w_{r}^{-} / w_{r}^{+}$equals $(1-P) / P$. Therefore, the latter is mainly a measure for the asymmetry of the relative velocity distributions. One can relate the curves of figure 11(b) to the distributions of figures 7 and 9 . The ratio $(1-P) / P$ merely represents the ratio of the probability of having negative and positive relative velocities. Therefore, the shape of 

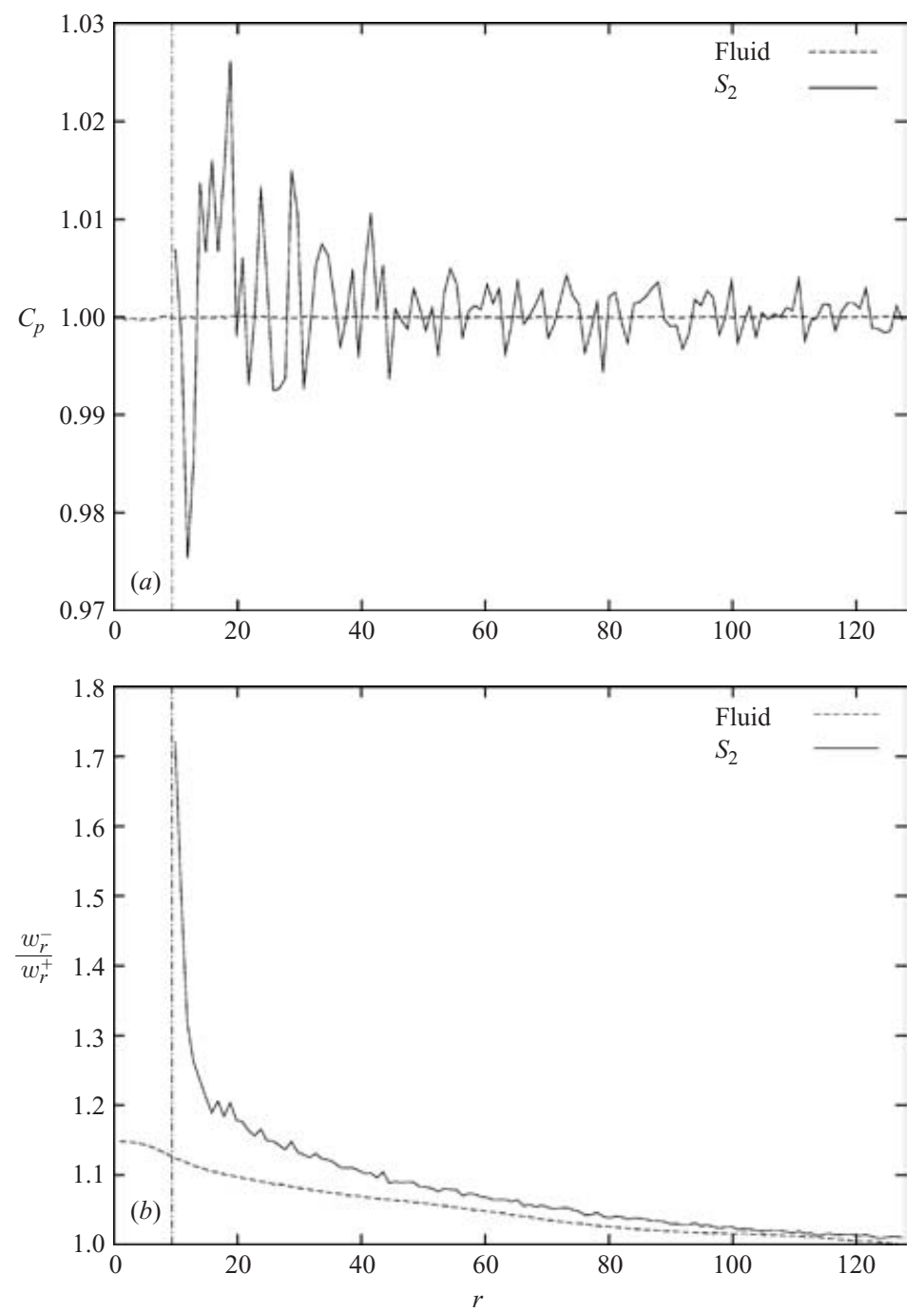

FIgURE 11. (a) The flux-balance coefficient $C_{p}$ and $(b)$ the ratio of $w_{r}^{-} / w_{r}^{+}$as a function of distance $r$. 'Fluid' indicates the single-phase simulation.

the curves in figure $11(b)$ can be considered as a characteristic feature of the turbulent (particle) velocity field. The strong increase of the ratio for the particle phase at close approach is then caused by the increased asymmetry of the particle-phase relative velocity p.d.f.

\subsection{Accumulation and preferential concentration of particles}

In direct numerical simulations of turbulent suspensions with the point-particle approach, the accumulation of inertial particles in regions of low vorticity and high strain rate has been observed by a number of authors (Squires \& Eaton 1990; Sundaram \& Collins 1997) and was studied in more detail by e.g. Reade \& Collins (2000) and Wang et al. (2000). This accumulation effect can have a large impact on the rate of collisions of particles. The above authors used the radial distribution function at contact, $g_{r}(R)$ (see also equation (5.10)), to quantify the accumulation 


$\begin{array}{llr} & g_{r}(R+\delta / 2) & \left\langle N_{\text {pairs }}\right\rangle \\ S_{1} & 2.70 \pm 0.36 & 59 \\ S_{2} & 2.80 \pm 0.14 & 497 \\ S_{3} & 2.98 \pm 0.10 & 1634 \\ S_{4} & 2.69 \pm 0.13 & 477 \\ S_{5} & 2.88 \pm 0.16 & 511\end{array}$

TABLE 6. $g_{r}(R)$ at $r=R+0.5$ and shell width $\delta=1$. The data were obtained from 75 particle field realizations. $\left\langle N_{\text {pairs }}\right\rangle$ indicates the average number of pairs per realization.

effect as a function of physical properties such as the Reynolds number and the particle relaxation time.

The radial distribution function $g_{r}(r)$ indicates the probability of observing a particle pair separated by a distance $r$, with respect to the probability of observing a particle pair in a uniformly distributed field. A residual radial distribution function is defined as $h_{r}(r) \equiv g_{r}(r)-1$. In figure 5, this accumulation effect can be observed most clearly in plots $(c)$ and $(d)$, i.e. at 5 and $10 \mathrm{vol} \%$ particles respectively.

In table 6, values of the radial distribution function near contact are given, determined at a bin width $\delta$ similar to the interval at which the relative velocity distributions in figure 9 were determined. The last column contains the average number of pairs counted in a single realization of the particle field and indicates the relatively low number of pairs at this bin width.

The usual explanation for the increase of $g_{r}(R)$ is that vortical structures in the flow field collect particles at their edge. It is likely that this mechanism is also present in our simulations, but since the separation of scales between the particle size and the integral length scale of the turbulence is only a factor 4 , this effect probably plays a weak role in our simulations.

A second mechanism for the increase in particle concentrations at short separations can be attributed to the influence of the short-range hydrodynamic interactions. In a study by Ladd (1997) on fully resolved settling spheres, a strong increase in the radial distribution function at short separations is also observed. In that study a similar approach for taking into account the lubrication force was used. As observed in the previous section, the relative radial velocity is reduced upon approach and a capturing mechanism occurs for particles with low relative velocities.

In table 6, trends in the dependence of $g_{r}$ on the simulation parameters can also be observed. Both an increase in particle concentration and an increase in particle inertia appear to enhance the accumulation effect, although these effects are smaller than the statistical accuracy. In previous studies by Sundaram \& Collins (1997) it was found that in the limit of low particle concentrations, the radial distribution function was practically independent of the volume fraction.

\subsection{Collision frequencies}

The simulations offer two different routes to determine the collision frequency and collision kernel. First, by recording the discrete collision events during the simulations, one can obtain a value for the collision kernel directly. Secondly, using the radial relative velocity distributions and the radial distribution function obtained from our preceding analysis, one can compute the collision kernel using equation (5.10). In this section the first approach will be discussed and then compared to the latter one. 
All collision events are recorded throughout the duration of the simulation. In the previous analysis it was observed that near contact, particle accumulation occurs and that the relative velocity distribution near contact had a strong increase towards low relative velocities. These effects are caused by the velocity correlation in the turbulent flow field at short separations and the short-range hydrodynamic forces acting in the gap between the particles. The latter may cause the particle pair to capture each other and stay in each others vicinity for some time, until the particles are separated again by the external flow field. As a result, the probability that a second collision between two particles occurs increases strongly. A particle pair may exhibit a number of collisions closely spaced in time until the particle pair is separated. If more than two particles form a cluster, three or more neighbouring particles can collide with each other collectively, which enhances the high-frequency collision rates. This image is supported by the collision events recorded during the simulations.

Sundaram \& Collins (1996) demonstrate that similar behaviour can occur for particles in a simple shear flow, even when lubrication forces are not present, because the post-collision trajectories of the particles coincide. In their analysis, the number of collisions that two particles have before they separate is determined by the particle inertia.

In our simulations we distinguish two types of collisions, designated primary and secondary collisions. The first type of collision takes place between two particles that approach each other from a relatively large separation, whose motion is dominated by the random large-scale structures in the flow field and therefore is practically uncorrelated. The resulting collision event can be considered a random process.

Once a first contact has been established, two particles may stay closely together for some time. In a physical system, the collective motion of the particle pair may be such that the two particles smoothly slide over each other, mediated by the action of the interstitial fluid between the particles, until again separated by the turbulent flow field. In our simulations, this close contact process can only be mimicked at discrete time steps. Consequently, collisions between two particles at high frequency are recorded during the simulations, which reflect this sliding contact process. These secondary collisions are therefore at least partially a reflection of the discretization of the particle motion and may be sensitive to the choices made in our numerical approach. For this reason we will primarily focus our discussion on the primary collisions.

One way to study the collision behaviour of the particles in the turbulent flow field is by observing the time between two collisions. To this end we study the probability density function (p.d.f.) of the time between two collisions. Uncorrelated particles move randomly and behave as a kinetic gas. The distribution of collision times for such a random process is described by an exponential distribution,

$$
P_{c}\left(t_{c}\right)=\frac{1}{\tau_{c}} \exp \left(-\frac{t_{c}}{\tau_{c}}\right)
$$

where $\tau_{c}$ is the average time between two collisions (see also equation (5.1)). The distribution of the collision times offers a statistical method to distinguish between the two types of collisions in our simulations.

In figure 12, the distribution of the time interval between two collisions of a particle with any other particle is given, as obtained from all particles during the run of a simulation. This distribution shows an exponential decay at long collision times and a sharp increase at short collision times. The exponential tail corresponds to primary collisions, where the time interval between two random collisions is exponentially 

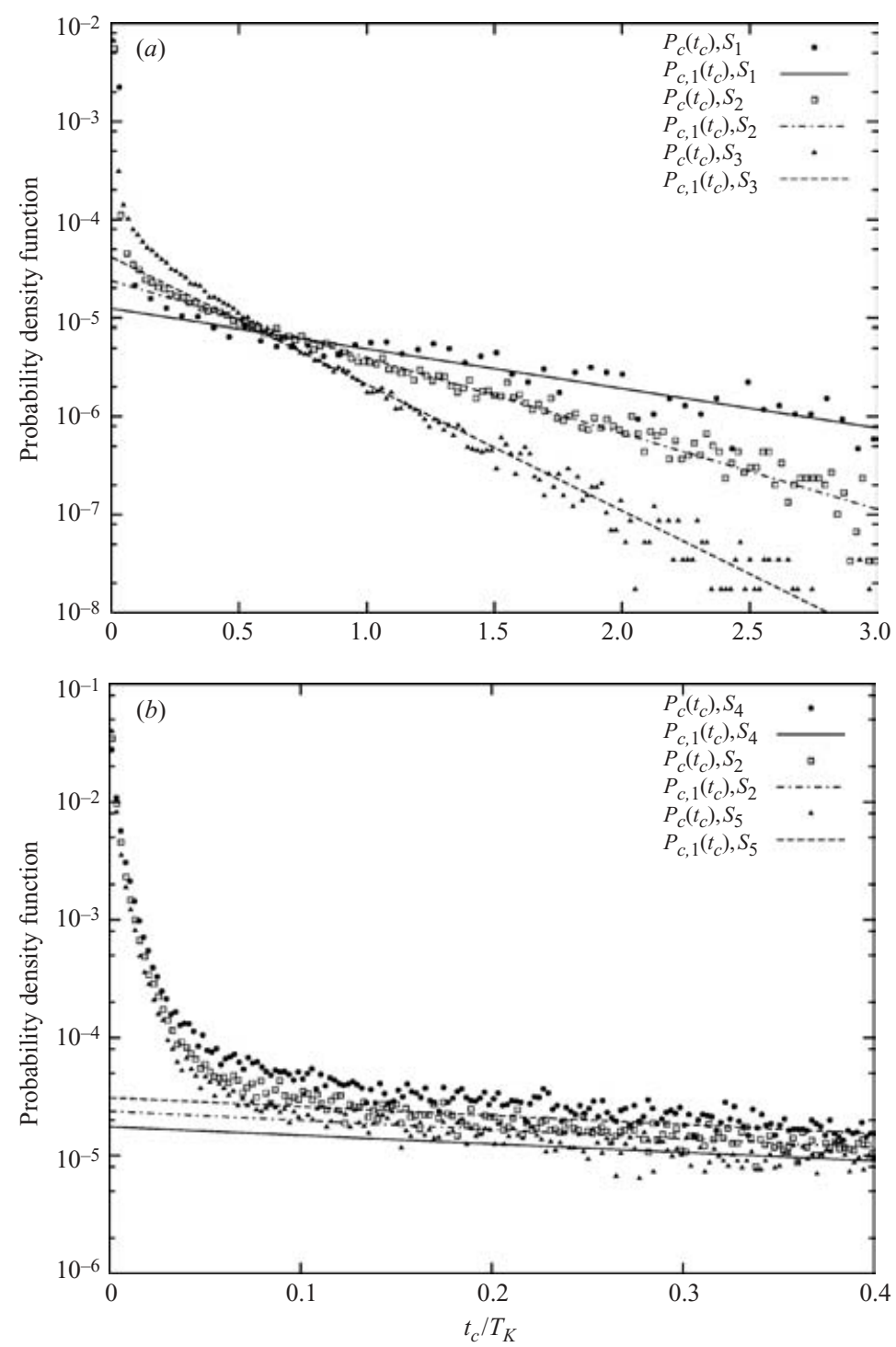

Figure 12. Probability density function of collision times. (a) Simulations $S_{1}$ to $S_{3}$ indicate the influence of volume concentration and $(b)$ simulations $S_{4}, S_{2}$ and $S_{5}$ demonstrate the influence of particle to fluid density ratio on the collision time p.d.f. The lines of $P_{c, 1}\left(t_{c}\right)$ relate to equations (5.13) and (5.14).

distributed. The large increase at short time intervals corresponds to secondary collisions.

To quantify the two different collision mechanisms, we propose the following form for the probability density function:

$$
P_{c}\left(t_{c}\right)=\alpha_{c} P_{c, 1}\left(t_{c}\right)+\left(1-\alpha_{c}\right) P_{c, 2}\left(t_{c}\right)
$$

where $P_{c, 1}$ is the probability of observing primary collisions, and $P_{c, 2}$ the probability of secondary collisions. $P_{c, 1}$ may be described by equation $(5.13)$. In this formulation, the collision process is characterized by three parameters: $\alpha_{c}$ is the fraction of primary 


\begin{tabular}{lccccccc}
\hline & $\alpha_{c}(\%)$ & $\tau_{c, 1} / T_{K}$ & $\tau_{c, A b} / T_{K}$ & $\tau_{c, 2} / T_{K}$ & $\Gamma_{c, 1}$ & $\Gamma_{c, A b}$ & $\Gamma_{c, s p h}$ \\
& $8.80 \pm 0.54$ & $1.08 \pm 0.07$ & 1.15 & 0.028 & 6.11 & 5.71 & 2.73 \\
$S_{1}$ & $8.79 \pm 0.44$ & $0.56 \pm 0.01$ & 0.41 & 0.025 & 4.12 & 5.58 & 2.72 \\
$S_{2}$ & $9.21 \pm 0.60$ & $0.34 \pm 0.01$ & 0.25 & 0.026 & 3.90 & 5.36 & 3.10 \\
$S_{3}$ & $6.95 \pm 0.30$ & $0.60 \pm 0.01$ & 0.40 & 0.025 & 3.85 & 5.73 & 2.58 \\
$S_{4}$ & $11.71 \pm 0.44$ & $0.57 \pm 0.01$ & 0.41 & 0.026 & 4.04 & 5.64 & 2.94 \\
$S_{5}$ & & &
\end{tabular}

TABLE 7. Characteristic collision time scales $\tau_{c}$ and collision kernels $\Gamma$ of suspension simulations $S_{1}$ to $S_{5} . \Gamma_{c, 1}$ was calculated from the primary collision times, $\Gamma_{c, A b}$ was calculated based on the model of Abrahamson (1975) and $\Gamma_{c, s p h}$ was based on the spherical formulation of the collision kernel.

collisions, $\tau_{c, 1}$ is the average collision time for primary collisions and $\tau_{c, 2}$ is the average time for secondary collisions. The parameters $\alpha_{c}$ and $\tau_{c, 1}$ can be determined by fitting the long-time tail of the distributions. After determination of these two parameters, $\tau_{c, 2}$ can be determined from $P_{c, 2}$ via

$$
\tau_{c, 2}=\int_{0}^{\infty} t_{c} P_{c, 2}\left(t_{c}\right) \mathrm{d} t_{c}=\int_{0}^{\infty} t_{c}\left(\frac{P_{c}\left(t_{c}\right)-\alpha P_{c, 1}\left(t_{c}\right)}{(1-\alpha)}\right) \mathrm{d} t_{c} .
$$

The curve fits representing $P_{c, 1}$ are presented in figure 12 while the parameter values are given in table 7 .

The results indicate that by describing the collision process according to equation (5.14), the influence of particle volume concentration can be separated from that of particle inertia. At increasing particle concentration, the collision time $\tau_{c, 1}$ decreases, which corresponds to a decrease in mean free path of the particles. This is clearly demonstrated in figure 12(a), where the slope of the tail is a function of the particle volume concentration. Table 7 indicates that $\alpha_{c}$ remains practically constant with varying volume fraction. When the particle inertia is varied at constant volume concentration (see figure 12b), the particle collision time is practically constant while $\alpha_{c}$ increases with increasing inertia. As particle inertia increases, the particles become less sensitive to the details of the collision process (lubrication force, velocity correlation or the numerical collision procedure), which favours the occurrence of primary collisions.

In table 7 , the time between primary collisions is compared with the theoretical model of Abrahamson (1975) that predicts the collision frequency of uncorrelated particles. The collision rate is calculated via equations (5.1) and (5.2), with $u_{p}^{\prime}$ determined from the particle kinetic energy. For low volume concentrations $\tau_{c, 1}$ corresponds well with Abrahamson's result, but as the volume concentration increases, the simulations show a systematically larger collision time. According to Abrahamson (1975) (equation (5.2)), the collision kernel is a function of the particle r.m.s. velocity, and not of volume concentration. Table 7 however suggests that this is not the complete picture and a correction on the collision kernel for primary collisions is required.

The collision kernel for primary collisions can also be compared to the collision kernel as computed via the spherical formulation of equation (5.10). This collision kernel, given in the last column of table 7, is computed based on the values for the radial distribution function in table 6 and the radial relative velocity distributions discussed in $\S 5.2$. The values of $\Gamma_{c, s p h}$ obtained via this route are of the same order of magnitude, but approximately half that of the values of $\Gamma_{c, 1}$. This difference may arise for a number of reasons. First, the chosen bin width $\delta$, set to $1[l u]$, smears out the effect of the increasing radial distribution function towards contact. Thus, the estimate 
of $g_{r}(R+0.5 \delta)$ will be smaller than the actual value of $g_{r}(R)$, which can easily differ by a factor 2 . Secondly, in determination of the radial velocity distribution, the action of the short-range hydrodynamic forces gives a large contribution of low values in the absolute mean relative velocity. As a result, computing $\Gamma_{c, s p h}$ based on $\left\langle\left|w_{r}\right|\right\rangle$ will result in a lower value for the collision kernel compared to $\Gamma_{c, 1}$, since the latter only describes the collision rate of particles that have uncorrelated motion, and therefore have a larger relative velocity. Thus the trends observed in the primary collision kernel are not present in $\Gamma_{c, s p h}$ since the two collision kernels describe different aspects of the particle collisions.

\section{Summary and conclusions}

A simulation approach has been developed for the study of dense turbulent solidliquid suspensions. Simulations have been performed of a sustained isotropic turbulent flow, using a lattice-Boltzmann scheme for the fluid flow simulation with a spectral forcing scheme for the generation of turbulent conditions. The suspension contained up to 3900 freely moving particles. The particle diameter spanned approximately 8 grid points, ensuring that the flow field around the particles was fully resolved. For particles approaching contact, a collision detection algorithm was used. This was combined with a subgrid model based on lubrication theory to compensate for the lack of resolution to compute the flow in the gap between two particles separated by less than the grid spacing. Five turbulent suspension simulations have been carried out at a Taylor Reynolds number of 61 , where the volume fraction of particles was chosen between $2 \%$ and $10 \%$ and where the particle to fluid density ratio was varied between 1.15 and 1.73. The results of these simulations have been characterized in two ways. First, the interaction between the particle phase and the turbulent flow field has been studied. Second, the behaviour of the particle phase was characterized by studying particle collisions and particle relative velocity distributions.

An increase of the kinetic energy at wavenumbers around the particle wavenumber indicated that particles generate fluid motion at scales of the order of the particle size. Due to the relative motion between the particles and the fluid, velocity gradients are generated near the particle surface that enhance the rate of energy dissipation at large wavenumbers and consequently suppress the kinetic energy in the spectrum at smaller wavenumbers. The decrease of kinetic energy and energy dissipation at small wavenumbers is in quantitative agreement with results obtained from numerical studies on two-way coupled suspensions where the solid phase was treated as point particles. It has been demonstrated that at a constant mass loading, the changes in the spectrum are much more sensitive to an increase in volume fraction of particles than to an increase in particle inertia.

The radial relative velocity distribution of the particles near contact showed a large tail for negative velocities and a strong increase at small velocities, while the distribution was strongly reduced at larger positive velocities. This distribution suggests the following image: Particles may approach at large velocities, but at close range there is a reduction in relative velocities due to the action of lubrication forces and velocity correlation in the turbulent flow field. Under action of the turbulent flow field or due to the particle inertia, collisions occur. As particles separate, the lubrication force becomes attractive. This explains the strong decay of the radial velocity distribution at positive values. As separation increases, the distribution of the radial relative velocity becomes Gaussian. The transverse relative velocity distribution remained practically Gaussian even at close separations. 
The magnitudes of the radial and transverse relative velocities of the particles show a $1-f(r)$ and $1-g(r)$ dependence on the separation $r$, where $f(r)$ and $g(r)$ are the longitudinal and transverse fluid velocity correlation functions. This demonstrates that the particle motion is strongly linked to the structure of the turbulent flow field. The particle relative velocity shows a stronger decay than that of the fluid phase as the separation reduces to zero. A check of the flux-balance assumption demonstrates that the particle field reaches a dynamic steady state since the ratio of inward to outward particle flux practically equals 1 .

The spatial distribution of the particles was further characterized by the radial distribution function, which indicated that an increase in particle concentration at short separations occurred. This increase in concentration is caused by both the familiar 'preferential concentration' effect and the short-range hydrodynamic interactions.

The collision time distribution rises steeply for short collision times and decays exponentially for longer times, indicating the presence of two separate collision mechanisms. An exponential decay corresponds to an uncorrelated random collision process, comparable to the behaviour of a kinetic gas. The peak for short collision times corresponds to repetitive contacts between particles in correlated motion. Collisions due to uncorrelated particle motion were designated primary collisions; collisions due to correlated motion were designated secondary collisions. The latter originate from a number of aspects such as short-range hydrodynamic interactions, spatial correlation in the turbulent flow field at short separations, and the numerical treatment of the lubrication forces and particle collisions in the simulations. In this respect there is room for improvement and a need for further evaluations, since the choices made in the subgrid forces and collision modelling may influence the short-range behaviour of the suspension simulations.

From a decomposition of the collision time distributions, two parameters were obtained to characterize primary collisions: the average collision time $\left(\tau_{c, 1}\right)$, and the fraction of primary collisions to the total number of collisions $\left(\alpha_{c}\right)$. An increase of the particle volume fraction decreased the average time between primary collisions. At low volume concentration the mean time corresponded well with kinetic theory for inertial particles at low volume concentrations (Abrahamson 1975) whereas at higher volume fraction, the average collision time appeared larger than predicted by kinetic theory. By increasing the particle inertia, the fraction of primary collisions $\alpha_{c}$ was found to increase, leaving $\tau_{c}$ practically unaffected. The value of the collision kernel for primary collisions was of the same order of magnitude but roughly twice as large as the collision kernel predicted using the spherical formulation. This difference is probably a result of both the rather low statistical accuracy at which the radial distribution function at contact could be calculated and the fact that the two collision kernels describe different aspects of the collision process.

Our method proves to be an efficient tool to obtain detailed insight into the behaviour of turbulent suspensions with a full recovery of the two-way interactions between the particle and the fluid phase. This simulation approach can be used to gain insight into for instance the collision behaviour of crystals in dense turbulent suspensions, such as encountered in industrial crysallization processes. In these processes, crystal collisions occurring at sufficiently high impact velocity can lead to fragmentation. Our simulations offer possibilities of studying the rate of fragmentation of crystals in dense turbulent suspensions, based on e.g. the radial velocity distribution near impact. 
The authors thank Professor John McLaughlin for kindly providing us with the collision detection algorithm used in this research.

\section{REFERENCES}

Abrahamson, J. 1975 Collision rates of small particles in a vigorously turbulent fluid. Chem. Engng Sci. 30, 1371-1379.

Aidun, C. K. \& Lu, Y. 1995 Lattice-Boltzmann simulation of solid particles. J. Statist. Phys. 81, 49-61.

Aidun, C. K., Lu, Y. \& Ding, E.-J. 1998 Direct analysis of particulate suspensions with inertia using the discrete boltzmann equation. J. Fluid Mech. 373, 287-311.

Alvelius, K. 1999 Random forcing of three-dimensional homogeneous turbulence. Phys. Fluids 11, $1880-1889$.

BEHREND, O. 1995 Solid-fluid boundaries in partcle suspension simulations via the lattice Boltzmann method. Phys. Rev. E 52 (1), 1164-1175.

Boivin, M., Simonin, O. \& Squires, K. D. 1998 Direct numerical simulation of turbulence modulation by particles in isotropic turbulence. J. Fluid Mech. 375, 235-263.

Bouzidi, M., Firdaouss, M. \& Lallemand, P. 2001 Momentum transfer of a boltzmann-lattice fluid with boudaries. Phys. Fluids 13, 3452-3459.

Brunk, B. K., Koch, D. L. \& Lion, L. W. 1998 Turbulent coagulation of colloidal particles. J. Fluid Mech. 364, 81-113.

Chen, H., Teixeira, C. \& Molvig, K. 1998 a Realization of fluid boundary conditions via discrete boltzmann dynamics. Intl J. Mod. Phys. C 9 (8), 1281-1292.

Chen, M., Kontomaris, K. \& Mclaughlin, J. $1998 b$ Direct numerical simulation of droplet collisions in a turbulent channel flow. Part I: Collision algorithm. Intl J. Multiphase Flow 24, 1079-1103.

Chen, S. \& Doolen, G. D. 1998 Lattice Boltzmann method for fluid flows. Annu. Rev. Fluid Mech. 30, 329-364.

Crowe, C., Sommerfeld, M. \& Tsuji, Y. 1997 Multiphase Flows with Droplets and Particles, 1st Edn. CRC Press.

Crowe, C. C. 2000 On models for turbulence modulation in fluid-particle flows. Intl J. Multiphase Flow 26, 719-727.

Derksen, J. J. \& Van den Akker, H. E. A. 1999 Large eddy simulations on the flow driven by a rushton turbine. AIChE J. 45, 209-221.

Derksen, J. J. \& VAN Den AkKer, H. E. A. 2000 Simulation of vortex core precession in a reverse-flow cyclone. AIChE J. 46, 1317-1331.

Ding, E.-J. \& Aidun, C. K. 2000 The dynamics and scaling law for particles suspended in shear flow with inertia. J. Fluid Mech. 423, 317-344.

Eggels, J. G. M. \& Somers, J. A. 1995 Numerical simulation of free convective flow using the lattice-Boltzmann scheme. Intl J. Heat Fluid Flow 16, 357-364.

Elghobashi, S. \& Truesdell, G. 1993 On the two-way interaction between homogenous turbulence and dispersed solid particles. I: Turbulence modification. Phys. Fluids 5, 1790-1801.

EsWARAN, V. \& POPE, S. 1988 An examination of forcing in direct numerical simulations of turbulence. Comput. Fluids 16, 257-278.

Felderhof, B. \& Ooms, G. 1989 Effective mass density of fluid suspensions. Phys. Fluids 1, 1091-1097.

Gahn, C. \& Mersmann, A. 1999 Brittle fracture in crystallization processes part a: Attrition and abrasion of brittle solids. Chem. Engng Sci. 54, 1273-1282.

Goldstein, D., Handler, R. \& Sirovich, L. 1993 Modeling a no-slip flow boundary with external force field. J. Comput. Phys. 105, 354-366.

Gondret, P., Hallouin, E., Lance, M. \& Petit, L. 1999 Experiments on the motion of a solid sphere toward a wall: From viscous dissipation to elastohydrodynamic bouncing. Phys. Fluids 11, 2803-2805.

Gondret, P., Lance, M. \& Petit, L. 2002 Bouncing motion of spherical particles in fluids. Phys. Fluids 14, 643-652. 
Наsimoто, Н. 1959 On the periodic fundamental solutions of the Stokes equations and their application to viscous flow past a cubic array of spheres. J. Fluid Mech. 5, 317-328.

He, X., Zou, Q., Luo, L.-S. \& Dembo, M. 1997 Analytic solutions of simple flows and analysis of nonslip boundary conditions for the lattice Boltzmann BGK model. J. Statist. Phys. 87, $115-136$.

HeEmels, M. 1999 Computer simulations of colloidal suspensions using an improved latticeboltzmann scheme. PhD thesis, Delft University of Technology.

HinZe, J. O. 1975 Turbulence, 2nd edn. McGraw-Hill.

Hollander, E., Derksen, J., Portela, L. \& Van den Akker, H. 2001 Numerical scale-up study for orthokinetic agglomeration in stirred vessels. AIChE J. 47, 2425-2440.

Hu, H. 1996 Direct simulation of solid-liquid mixtures. Intl J. Multiphase Flow 22, 335-352.

Hu, H. H., Patankar, N. A. \& Zhu, M. Y. 2001 Direct numerical simulations of fluid-solid systems using the arbitrary Lagrangian-Eulerian technique. J. Comput. Phys. 169, 4217-462.

Joseph, G., Zenit, R., Hunt, M. \& Rosenwinkel, A. 2001 Particle-wall collisions in a viscous fluid. J. Fluid Mech. 433, 329-346.

Kim, S. \& Karrila, S. J. 1991 Microhydrodynamics: Principles and Selected Applications. Butterworth-Heinemann.

Косн, D. L. \& Hill, R. J. 2001 Inertial effects in suspension and porous-media flows. Annu. Rev. Fluid Mech. 33, 619-647.

Kruis, F. \& Kusters, K. 1997 The collision rate of particles in turbulent flow. Chem. Engng Commun. 158, 201-230.

LADD, A. J. C. 1994a Numerical simulations of particulate suspensions via a discretized boltzmann equation. Part 1. Theoretical foundation. J. Fluid Mech. 271, 285-309.

LADD, A. J. C. $1994 b$ Numerical simulations of particulate suspensions via a discretized boltzmann equation. Part 2. Numerical results. J. Fluid Mech. 271, 311-339.

LAdD, A. J. C. 1997 Sedimentation of homogeneous suspensions of non-brownian spheres. Phys. Fluids 9, 491-499.

LADD, A. J. C. 2002 Effects of container walls on the velocity fluctuations of sedimenting spheres. Phys. Rev. Lett. 88, 04830-1 04830-4.

Ladd, A. J. C. \& Verberg, R. 2001 Lattice-Boltzmann simulations of particle-fluid suspensions. J. Statist. Phys. 104, 1191-1251.

LAI, M.-C. \& PESKIN, C. S. 2000 An immersed boundary method with formal second-order accuracy and reduced numerical viscosity. J. Comput. Phys. 160, 705-719.

Maury, B. 1997 A many-body lubrication model. C.R. Acad. Sci. Paris I 325, 1053-1058.

MeI, R., Luo, L.-S. \& ShyY, W. 1999 An accurate curved boundary treatment in the lattice Boltzmann method. J. Comput. Phys. 155, 307-330.

Moin, P. \& Mahesh, K. 1998 Direct numerical simulation: A tool in turbulence research. Аnnu. Rev. Fluid Mech. 30, 539-578.

Ooms, G. \& JANSEN, G. 2000 Particles-turbulence interaction in stationary, homogeneous, isotropic turbulence. Intl J. Multiphase Flow 26, 1831-1850.

Overholt, M. \& Pope, S. 1998 A deterministic forcing scheme for direct numerical simulations of turbulence. Comput. Fluids 27, 11-28.

PAN, Y. \& BANERJEe, S. 1997 Numerical investigation of the effects of large particles on wallturbulence. Phys. Fluids 9, 3786-3807.

Patankar, N. A. 2001 A formulation for fast computations of rigid particulate flows. Annu. Res. Briefs, Center for Turbulence Research, Stanford University.

Patankar, N. A., Singh, P., Joseph, D., Glowinski, R. \& Pan, T.-W. 2000 A new formulation of the distributed Lagrange multiplier/fictitious domain method for particulate flows. Intl $J$. Multiphase Flow 26, 1509-1524.

QI, D. 1999 Lattice-Boltzmann simulation of particles in non-zero-Reynolds-number flows. J. Fluid Mech. 385, 41-62.

Reade, W. C. \& Collins, L. R. 2000 Effect of preferential concentration on turbulent collision rates. Phys. Fluids 12, 2530-2540.

Rohde, M., Derksen, J. \& VAn den Akker, H. E. A. 2002 Volumetric method for calculating the flow around moving objects in lattice-Boltzmann schemes. Phys. Rev. E 65, 056701.

Rothman, D. H. \& ZaLeski, S. 1997 Lattice-Gas Cellular Automata, 1st Edn. Cambridge University Press. 
Saffman, P. \& Turner, J. 1956 On the collision of drops in turbulent clouds. J. Fluid Mech. 1, $2530-2540$.

Sommerfeld, M. 2001 Validation of stochastic Lagrangian modelling approach for inter-particle collisions inhomogeneous isotropic turbulence. Intl J. Multiphase Flow 27, 1829-1858.

Squires, K. D. \& Eaton, J. K. 1990 Particle response and turbulence modification in isotropic turbulence. Phys. Fluids 2, 1191-1203.

Sundaram, S. \& Collins, L. R. 1996 Numerical considerations in simulating a turbulent suspension of finite-volume particles. J. Comput. Phys. 124, 337-350.

Sundaram, S. \& Collins, L. R. 1997 Collision statistics in an isotropic particle-laden turbulent suspension. Part 1. Direct numerical simulations. J. Fluid Mech. 335, 75-109.

Sundararajakumar, R. R. \& Koch, D. L. 1996 Non-continuum lubrication flows between particles colliding in a gas. J. Fluid Mech. 313, 283-308.

Ten Cate, A., Derksen, J. J., Kramer, H. J. M., Van Rosmalen, G. M. \& Van den Akker, H. E. A. 2001 The microscopic modelling of hydrodynamics in industrial crystallisers. Chem. Engng Sci. 56, 2495-2509.

Ten Cate, A., Nieuwstad, C. H., Derksen, J. J. \& Van den Akker, H. E. A. 2002 PIV experiments and lattice-Boltzmann simulations on a single sphere settling under gravity. Phys. Fluids 14, 4012-4025.

Verberg, R. \& LADD, A. 2002 Accuracy and stability of a lattice-Boltzmann model with subgrid scale boundary conditions. Phys. Rev. E 65, 016701-1-16.

Wang, L.-P., WeXler, A. S. \& Zhou, Y. 1998 Statistical mechanical descriptions of turbulent coagulation. Phys. Fluids 10, 2647-2651.

WANG, L.-P., WeXler, A. S. \& Zhou, Y. 2000 Statistical mechanical description and modelling of turbulent collision of inertial particles. J. Fluid Mech. 415, 117-153.

Wibowo, C. \& NG, K. M. 2001 Operational issues in solids processing plants: Systems view. AIChE J. 47, 107-125.

YeUNG, P. \& ZHOU, Y. 1997 Universality of the kolmogorov constant in numerical simulations of turbulence. Phys. Rev. E 56 (2), 1746-1752.

Yuan, Z. \& Michaelides, E. 1992 Turbulence modulation in particulate flows - a theoretical approach. Intl J. Multiphase Flow 18, 779-785.

YUU, S. 1984 Collision rate of small particles in a homogeneous isotropic turbulence. AIChE J. 30, 802-807.

Zwietering, T. N. 1958 Suspending of solid particles in liquid agitators. Chem. Engng Sci. 8, $244-253$. 